\title{
Synthesis and Antiproliferative Activity of 3 and 7-Styrylcoumarins
}

Angie Herrera ${ }^{\dagger a, b}$, Wilson Castrillón ${ }^{\dagger a} \bullet$ Elver Otero $^{\mathrm{a}} \bullet$ Esneyder Ruiz $^{\mathrm{a}}$, Miguel Carda $^{\mathrm{c}}$, Raúl Agut $^{\mathrm{c}}$, Tonny Naranjo ${ }^{\mathrm{d}, \mathrm{e}}$, Gustavo Moreno ${ }^{\mathrm{a}, \mathrm{b}}$, Maria Elena Maldonado ${ }^{\mathrm{b} *}$ and Wilson Cardona-G ${ }^{\mathrm{a}^{*}}$

a. Química de Plantas Colombianas, Institute of Chemistry, Faculty of Exact and Natural Sciences, University of Antioquia UdeA, Calle 70 No. 52-21, A.A 1226, Medellín, Colombia.

b. Grupo Impacto de los Componentes Alimentarios en la Salud, School of Dietetics and Human Nutrition, University of Antioquia, A.A. 1226 Medellín.

c. Department of Inorganic and Organic Chemistry, University Jaume I, E-12071 Castellón, Spain.

d. Grupo de Micología Médica y Experimental, Corporación para Investigaciones Biológicas, Medellín, Colombia

e. School of Health Sciences, Universidad Pontificia Bolivariana, Medellín, Colombia

*Author to whom correspondence should be addressed; e-mail: wilson.cardona1@udea.edu.co (W.C); maria.maldonado@udea.edu.co

$\dagger$ Both authors contributed equally to this work.

\begin{abstract}
A series of styrylcoumarins were obtained via Mizoroki-Heck reactions between 3-bromo-4-methyl7-(octyloxy)-2H-chromen-2-one or 2-oxo- $2 H$-chromen-7-yl trifluoromethanesulfonate and functionalized styrenes. The structures of the products were elucidated by spectroscopic analysis. All compounds were evaluated against SW480 and CHO-K1 cell lines. A number of hybrids showed good antiproliferative activity. Among the tested compounds, hybrids 6e, 10c and 10d, exhibited the highest activity ( $\mathrm{IC}_{50-} \mathrm{SW} 480 / 48 \mathrm{~h}=6,92 ; 1,01$ and $5,33 \mu \mathrm{M}$, respectively) and selectivity $\left(\mathrm{IS}_{48 \mathrm{~h}}=>400 ; 67,8\right.$ and 7,2, respectively). In addition, these compounds were able to preserve their activities over time. The results achieved by these hybrids were even better than the lead compounds (coumarin and resveratrol) and the standard drug (5-FU). As regards structure-activity relationship it seems that the location of the styryl group on the coumarin structure and the presence of the hydroxyl group on the phenyl ring were determinant for the activity.
\end{abstract}

Keywords: coumarin, styrylcoumarin, antiproliferative activity, colorectal cancer

Abbreviations: 5-FU: 5-Fluorouracil; CRC: Colorectal Cancer; DMSO: dimethylsulfoxide; NF- $\kappa B$ : Nuclear factor- $\kappa \mathrm{B}$. 


\section{Introduction}

Colorectal cancer (CRC) is the third most commonly diagnosed malignancy and the fourth leading cause of cancer death worldwide (Arnold et al., 2016). This pathology presents a great geographical distribution and the patterns are very similar among men and women, being the third most common cancer in men and the second one in women (Globocan, 2012).

Current treatments for CRC include combinations of chemotherapeuthic agents such as FOLFOX (5-FU/leucovorin/oxaliplatin) and FOLFIRI (folic acid/5-FU/irinotecan) which are composed of 5fluorouracil as the backbone of treatment, that, although effective, cause undesirable gastrointestinal and neurological side-effects which many times result in dose limitations or cessation of the anticancer therapy (McQuade et al., 2014; Pointet \& Taieb, 2017). Thus, it becomes necessary to discover new, more potent, and selective agents for treating this disease.

In this regard, many pharmacological studies dealing with coumarins as a key structural motif have been reported. The pharmacological actions with which coumarins are endowed include anti-HIV (Kashman 1992), anti-inflammatory (Resch et al., 1998), anticoagulant (Madari et al., 2003), antimicrobial (Ankleka et al., 2003), antiplatelet (Vilar et al., 2006), antiprotozoal (Arango et al., 2010; Pierson et al., 2010) and antioxidant (Gacche et al., 2012). Besides, some coumarins have exhibited antimycobacterial activity which is potentiated when they bear a fatty acid residue in their structure (Yee et al., 2005). Furthermore, coumarins have also shown inhibition of cell proliferation in different types of cancer cell lines (Egan et al., 1997; Kawaii et al., 2001; Budzisz et al., 2003; Yang et al., 2006; Riveiro et al., 2008). According to this, some coumarin antibiotics can bind to the carboxyl terminus of Hsp90 molecular chaperone, which modulate multiple oncogenic pathways and is overexpressed in human malignancies, causing its inhibition (Neckers et al., 2002; Zhao et al., 2011). On the other hand, natural product resveratrol, which belongs to the family of compounds known as stilbenes, has great significance because of its wide variety of biological properties (Baur et al., 2006; Kedzierski et al., 2007; Udenigwe et al., 2008; Gülçin et al., 2010), including among them anticancer activity (Borriello et al., 2014). Resveratrol inhibits CRC in all phases acting on different targets such as NF- $\mathrm{KB}$, AP-1, cyclooxygenase and kinases (Athar et al., 2009). This compound enhances the activation of tumor suppressor p53 which ultimately induces apoptosis of human colon cancer cells. It also activates caspases 3 and 8 and increases the BAX/Bcl-2 ratio (Temraz et al., 2013).

The combination of two pharmacological agents into a single molecule, which leads to what is known as a hybrid molecule, has resulted in an emerging strategy in medicinal chemistry and drug discovery research (Meunier, 2008). These hybrid molecules may display dual activity, but 
do not necessarily act on the same biological target (Tsogoeva, 2010, atención este review sobre moléculas híbridas es más reciente que el de 2010, quizá sea interesant incluirlo también: Shaveta, Mishra, S, Singh. P (2016) Hybrid molecules: The privileged scaffolds for various pharmaceuticals. Eur J Med Chem 124: 500-536). Combining chemotherapy with agents having different mechanisms of action is one of the methods adopted for treating cancer (Mayur et al., 2009; Solomon et al., 2009). Several stilbene-coumarin hybrids were identified as very promising candidates by their excellent antiproliferative potency or by their remarkable apoptosis-inducing activity (see fig. 1). Compound $\mathbf{1 a}$ and $\mathbf{1 b}$ showed high activity against lung carcinoma H460 with $\mathrm{IC}_{50}$ values of 0.45 and $0.29 \mu \mathrm{M}$, respectively (Belluti et al., 2010). Styrylcoumarin 1c was evaluated for its antitumor activity against MCF-7 and HCT-28 tumor cell lines. This compound showed varying degrees of growth inhibition on the above mentioned cell lines with $\mathrm{IC}_{50}$ values of 7.32 and $3.78 \mu \mathrm{M}$, respectively (Shen et al., 2013). 3-Phenylcoumarin 1d exhibited potent antiproliferative activity with IC $_{50}$ values of 5.2 and $7.5 \mu \mathrm{M}$ against HL-60 and A549 cell lines, respectively (Yang et al., 2011). Benzimidazole-coumarin hybrid 1e was screened for in vitro antitumor activity on different cell lines. This compound, tested at a concentration of $10 \mu \mathrm{M}$, induced more than $50 \%$ inhibition of most of the cell lines, with higher selectivity against leukemic cancer cell lines (Paul et al., 2013). Coumarin-chalcone hybrid 1f showed around 30 fold more selectivity towards C33A (cervical carcinoma) cells over normal fibroblast NIH3T3 cells with an $\mathrm{IC}_{50}$ value of $3.59 \mu \mathrm{M}$ (Sashidhara et al., 2010). The resveratrol-imidazole hybrid $1 \mathrm{~g}$ showed cytotoxic activity against leukemia and renal cancer cell lines (4.1 and $8.3 \mu \mathrm{M}$, respectively) (Bellina et al., 2015). Resveratrol-chalcone conjugate $\mathbf{1 h}$ showed high selectivity towards certain ovarian cancer, non-small cell lung cancer and breast cancer cell lines, with $\mathrm{GI}_{50}$ values in the range of 1.28-34.1 $\mu \mathrm{M}$ (Kumar et al., 2014).
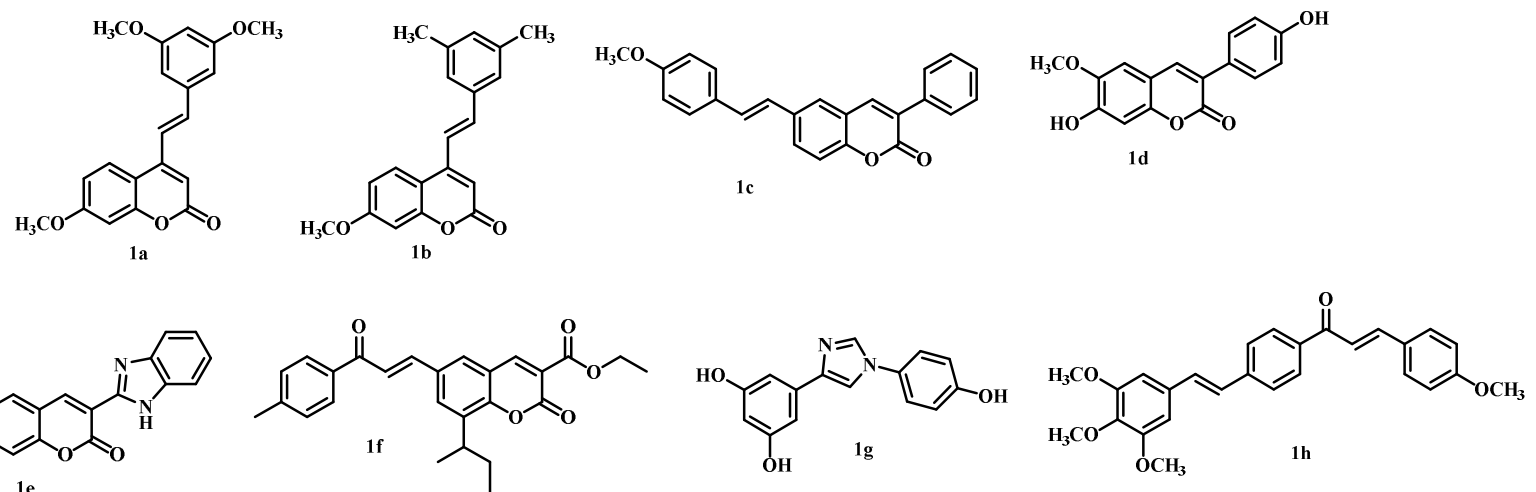

Fig. 1. Hybrid compounds endowed with antitumor activity 
Hybridizing the coumarin nucleus with other moieties has afforded new molecules with improved anticancer activity profile (Agarwal, 2000). In the search for new therapeutic alternatives to treat colorectal cancer, we have designed and synthesized a series of stilbene-coumarin hybrids (styrylcoumarins) (fig. 2). In addition, the anti-cancer activity of our hybrid molecules have been evaluated in vitro.
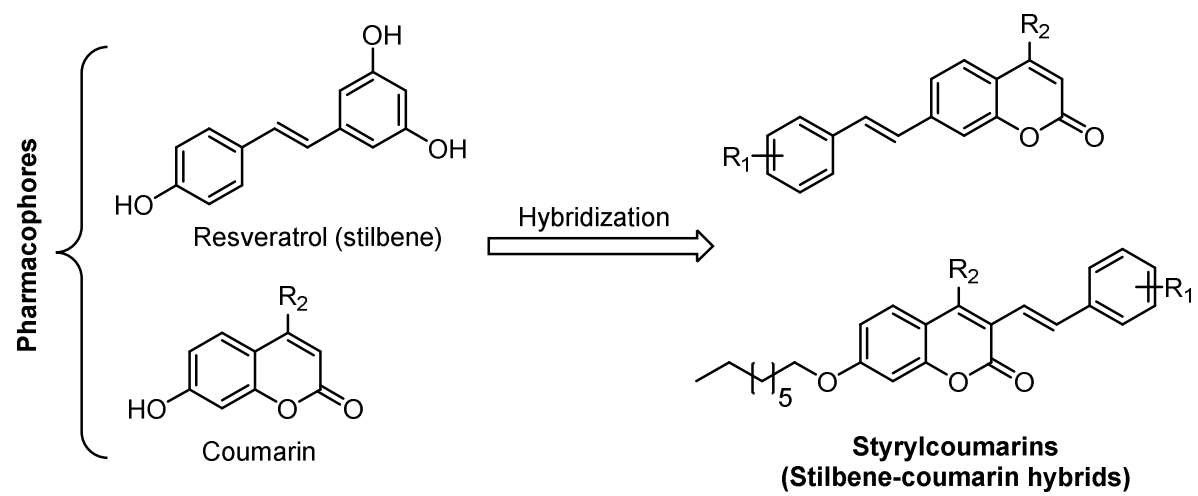

Fig. 2. Design of stilbene-coumarin hybrids as anti-cancer agents.

\section{Materials and Methods}

\section{Chemistry}

Microwave reactions were carried out in a CEM Discover microwave reactor in sealed vessels (monowave, maximum power $300 \mathrm{~W}$, temperature control by IR sensor, fixed temperature). ${ }^{1} \mathrm{H}$ and ${ }^{13} \mathrm{C}$ NMR spectra were recorded on a Varian instrument operating at 500 (300) and $125(75) \mathrm{MHz}$, respectively. The signals of the deuterated solvent $\left(\mathrm{CDCl}_{3}\right)$ were used as reference $\left(\mathrm{CDCl}_{3}: \delta=7.27\right.$ ppm for ${ }^{1} \mathrm{H}$ NMR and $\delta=77.00 \mathrm{ppm}$ for ${ }^{13} \mathrm{C}$ NMR). Carbon atom types $\left(\mathrm{C}, \mathrm{CH}, \mathrm{CH}_{2}, \mathrm{CH}_{3}\right)$ were determined by using the DEPT or APT pulse sequence. Signals were assigned using twodimensional heteronuclear correlations (COSY and HSQC). High resolution mass spectra were recorded using electrospray ionization mass spectrometry (ESI-MS). A QTOF Premier instrument with an orthogonal Z-spray-electrospray interface (Waters, Manchester, UK) was used operating in the $\mathrm{W}$-mode. The drying and cone gas was nitrogen set to flow rates of 300 and $30 \mathrm{~L} / \mathrm{h}$, respectively. Methanol sample solutions (ca. $1 \times 10^{-5} \mathrm{M}$ ) were directly introduced into the ESI spectrometer at a flow rate of $10 \mu \mathrm{L} / \mathrm{min}$. A capillary voltage of $3.5 \mathrm{kV}$ was used in the positive scan mode, and the cone voltage set to $U \mathrm{c}=10 \mathrm{~V}$. For accurate mass measurements, a $2 \mathrm{mg} / \mathrm{L}$ standard solution of leucine enkephalin was introduced via the lock spray needle at a cone voltage set to $85 \mathrm{~V}$ and a flow rate of $30 \mu \mathrm{L} / \mathrm{min}$. IR spectra were recorded on a Spectrum RX I FT-IR 
system (Perkin-Elmer, Waltham, MA, USA) in KBr disks. Silica gel 60 (0.063-0.200 mesh, Merck, Whitehouse Station, NJ, USA) was used for column chromatography, and precoated silica gel plates (Merck 60 F254 $0.2 \mathrm{~mm}$ ) were used for thin layer chromatography (TLC). Monitoring of the reaction progress and product purification was carried out by TLC.

Synthesis of 4-methyl-7-(octyloxy)-2H-chromen-2-one (3): Coumarin (1g, $5.7 \mathrm{mmol})$, potassium hydroxide $(955 \mathrm{mg}, 17 \mathrm{mmol})$ and acetonitrile $(20 \mathrm{~mL})$ were placed in a $50 \mathrm{~mL}$ flat-bottomed flask equipped with a magnetic stirring bar. The mixture was stirred and heated to reflux for a period of 5 min. Then, 1-bromooctane $(1.1 \mathrm{~mL}, 1.22 \mathrm{~g}, 6.3 \mathrm{mmol})$ was added to the reaction mixture which was then refluxed for $6 \mathrm{~h}$. The crude reaction mixture was concentrated on a rotatory evaporator and the residue was purified by column chromatography over silica gel. Elution with hexane and a mixture of hexane-ethyl acetate (9:1 ratio) gave rise to compound 3 (1.4 g, 85\% yield).

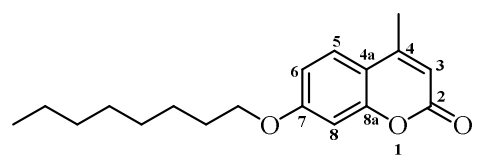

M.p. $48-50^{\circ} \mathrm{C}$; IR $\left(\mathrm{KBr}, \mathrm{cm}^{-1}\right): v \max 2924(\mathrm{C}-\mathrm{H}), 1718(\mathrm{C}=\mathrm{O}), 1620(\mathrm{C}=\mathrm{C}), 1512\left(\mathrm{C}=\mathrm{C}_{\mathrm{Ar}}\right), 1294(\mathrm{C}-$ O-C), 1199 ((C=O)-O), $839\left(\mathrm{C}-\mathrm{H}_{\mathrm{Ar}}\right) .{ }^{1} \mathrm{H} \mathrm{NMR}\left(\mathrm{CDCl}_{3}, 300 \mathrm{MHz}\right): \delta 0.93\left(\mathrm{CH}_{3}, \mathrm{t}, J=6.5 \mathrm{~Hz}\right), 1.35$ $\left(3 \mathrm{CH}_{2}, \mathrm{~m}\right), 1.51\left(\mathrm{CH}_{2}, \mathrm{~m}\right), 1.64\left(\mathrm{CH}_{2}, \mathrm{~m}\right), 1.86\left(\mathrm{CH}_{2}, \mathrm{~m}\right), 2.44\left(\mathrm{CH}_{3}, \mathrm{~s}\right), 4.05\left(-\mathrm{CH}_{2} \mathrm{O}-, \mathrm{t}, J=6.5\right.$ $\mathrm{Hz}), 6.17\left(\mathrm{H}_{3}, \mathrm{~s}\right), 6.85\left(\mathrm{H}_{8}, \mathrm{~d}, J=2.3 \mathrm{~Hz}\right), 6.90\left(\mathrm{H}_{6}, \mathrm{dd}, J=8.7,2.3 \mathrm{~Hz}\right) 7.53\left(\mathrm{H}_{5}, \mathrm{~d}, J=8.7 \mathrm{~Hz}\right) .{ }^{13} \mathrm{C}$ NMR $\left(\mathrm{CDCl}_{3}, 75 \mathrm{MHz}\right): \delta 14.11\left(\mathrm{CH}_{3}\right), 18.70\left(\mathrm{CH}_{3}\right) 22.68\left(\mathrm{CH}_{2}\right), 26.01\left(\mathrm{CH}_{2}\right), 28.99\left(\mathrm{CH}_{2}\right), 29.24$ $\left(\mathrm{CH}_{2}\right), 29.34\left(\mathrm{CH}_{2}\right), 31.80\left(\mathrm{CH}_{2}\right), 68.65\left(-\mathrm{OCH}_{2}\right), 101.34\left(\mathrm{C}_{8}\right), 111.82\left(\mathrm{C}_{6}\right), 112.74\left(\mathrm{C}_{3}\right), 113.40$ $\left(\mathrm{C}_{4 \mathrm{a}}\right), 125.46\left(\mathrm{C}_{5}\right), 152.63\left(\mathrm{C}_{8 \mathrm{a}}\right), 155.32\left(\mathrm{C}_{4}\right), 161.47(\mathrm{C}=\mathrm{O}), 162.29\left(\mathrm{C}_{7}\right)$. EIMS: m/z 289.1800 [M $+\mathrm{H}]^{+}$, (calculated for $\left.\left[\mathrm{C}_{18} \mathrm{H}_{25} \mathrm{O}_{3}\right]^{+}: 289.1804\right)$.

Synthesis of 3-bromo-4-methyl-7-(octyloxy)-2H-chromen-2-one (4): A solution of alkyloxycoumarin $3(1 \mathrm{~g}, 3.5 \mathrm{mmol})$ and NBS $(623 \mathrm{mg}, 3.5 \mathrm{mmol})$ in methanol $(20 \mathrm{~mL})$ was stirred in an ice bath. Then, $p$ - TsOH $(170 \mathrm{mg}, 1.0 \mathrm{mmol})$ in methanol $(10 \mathrm{~mL})$ was added to the reaction mixture for 10 min. Then, the reaction mixture was stirred at room temperature for $1 \mathrm{~h}$. The crude reaction mixture was concentrated on a rotatory evaporator and the residue was purified by column chromatography over silica gel. Elution with hexane and a mixture of hexane-ethyl acetate (95:5 ratio) afforded compound 4 (0.96 g, 75\% yield).

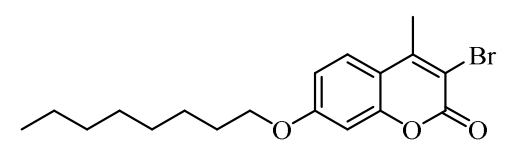


Yellow oil; IR (KBr, cm $\left.{ }^{-1}\right): v_{\max } 2924(\mathrm{C}-\mathrm{H}), 1737(\mathrm{C}=\mathrm{O}), 1614(\mathrm{C}=\mathrm{C}), 1468\left(\mathrm{C}=\mathrm{C}_{\mathrm{Ar}}\right), 1285(\mathrm{C}-\mathrm{O}-$ C), $1201((\mathrm{C}=\mathrm{O})-\mathrm{O}), 811\left(\mathrm{C}-\mathrm{H}_{\mathrm{Ar}}\right) ;{ }^{1} \mathrm{H} \mathrm{NMR}\left(\mathrm{CDCl}_{3}, 300 \mathrm{MHz}\right): \delta 0.93\left(\mathrm{CH}_{3}, \mathrm{t}, J=6.5 \mathrm{~Hz}\right), 1.35$ $\left(3 \mathrm{CH}_{2}, \mathrm{~m}\right), 1.51\left(\mathrm{CH}_{2}, \mathrm{~m}\right), 1.74\left(\mathrm{CH}_{2}, \mathrm{~m}\right), 1.86\left(\mathrm{CH}_{2}, \mathrm{~m}\right), 2.63\left(\mathrm{CH}_{3}, \mathrm{~s}\right), 4.05\left(-\mathrm{CH}_{2} \mathrm{O}-, \mathrm{t}, J=6.5\right.$ $\mathrm{Hz}), 6.84\left(\mathrm{H}_{8}, \mathrm{~d}, J=2.5 \mathrm{~Hz}\right), 6.92\left(\mathrm{H}_{6}, \mathrm{dd}, J=8.9,2.5 \mathrm{~Hz}\right), 7.53\left(\mathrm{H}_{5}, \mathrm{~d}, J=8.9 \mathrm{~Hz}\right) .{ }^{13} \mathrm{C} \mathrm{NMR}$ $\left(\mathrm{CDCl}_{3}, 75 \mathrm{MHz}\right): \delta 14.13\left(\mathrm{CH}_{3}\right), 19.50\left(\mathrm{CH}_{3}\right) 22.66\left(\mathrm{CH}_{2}\right), 25.98\left(\mathrm{CH}_{2}\right), 28.97\left(\mathrm{CH}_{2}\right), 29.23$ $\left(\mathrm{CH}_{2}\right), 29.33\left(\mathrm{CH}_{2}\right), 31.81\left(\mathrm{CH}_{2}\right), 68.79\left(-\mathrm{OCH}_{2}\right), 101.17\left(\mathrm{C}_{8}\right), 109.50\left(\mathrm{C}_{3}\right), 113.20\left(\mathrm{C}_{6}\right), 113.42$ $\left(\mathrm{C}_{4 \mathrm{a}}\right), 126.01\left(\mathrm{C}_{5}\right), 151.23\left(\mathrm{C}_{4}\right), 153.63\left(\mathrm{C}_{8 \mathrm{a}}\right), 157.48\left(\mathrm{C}_{7}\right), 162.37(\mathrm{C}=\mathrm{O})$. EIMS: m/z 367.0908 [M $+\mathrm{H}]^{+}$, (calculated for $\left[\mathrm{C}_{18} \mathrm{H}_{24} \mathrm{BrO}_{3}\right]^{+}:$367.0909).

Synthesis of 2-oxo-2H-chromen-7-yl trifluoromethanesulfonate (9): A solution of 7hydroxycoumarin $(\mathbf{8})(1.0 \mathrm{~g}, 6.17 \mathrm{mmol})$ in pyridine $(5 \mathrm{~mL})$ was cooled to $0^{\circ} \mathrm{C}$ and treated with triflic anhydride $(2.1 \mathrm{~mL}, 12.34 \mathrm{mmol}, 20 \mathrm{~min}$ addition). The reaction mixture was allowed to warm to room temperature and stirred for an additional $2.5 \mathrm{~h}$. The crude mixture was partitioned between saturated aqueous $\mathrm{CuSO}_{4}$ and ethyl acetate. After separation of the fractions, the organic fraction was dried $\left(\mathrm{Na}_{2} \mathrm{SO}_{4}\right)$ and concentrated under reduced pressure. The crude product was purified by column chromatography over silica gel. Elution with a mixture of hexane-ethyl acetate (8:2 ratio) afforded compound 9 in $85 \%$ yield (1.54 $\mathrm{mg}, 85 \%$ yield).

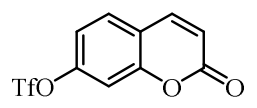

M.p. 78-80 ${ }^{\circ} \mathrm{C}$; IR $\left(\mathrm{KBr}, \mathrm{cm}^{-1}\right): v_{\max } 1720(\mathrm{C}=\mathrm{O}), 1421$ y $1220 \mathrm{~S}(=\mathrm{O})_{2}, 1130\left(\mathrm{CF}_{3}\right), 1294(\mathrm{C}-\mathrm{O}-\mathrm{C})$, $1110((\mathrm{C}=\mathrm{O})-\mathrm{O}), 748-979(\mathrm{C}-\mathrm{O}-\mathrm{S}) .{ }^{1} \mathrm{H} \mathrm{NMR}\left(\mathrm{CDCl}_{3}, 300 \mathrm{MHz}\right): \delta 6.53\left(\mathrm{H}_{3}, \mathrm{~d}, J=9.7 \mathrm{~Hz}\right), 7.25$ $\left(\mathrm{H}_{6}, \mathrm{dd}, J=2.3,8.6 \mathrm{~Hz}\right), 7.31\left(\mathrm{H}_{8}, \mathrm{~d}, J=2.3 \mathrm{~Hz}\right), 7.62\left(\mathrm{H}_{5}, \mathrm{~d}, J=8.6 \mathrm{~Hz}\right), 7.76\left(\mathrm{H}_{4}, \mathrm{~d}, J=9.7 \mathrm{~Hz}\right)$. ${ }^{13} \mathrm{C} \mathrm{NMR}\left(\mathrm{CDCl}_{3}, 75 \mathrm{MHz}\right): \delta 110.60\left(\mathrm{C}_{8}\right), 117.81\left(\mathrm{C}_{3}, \mathrm{C}_{6}\right), 118.66\left(-\mathrm{SO}_{3} \mathrm{CF}_{3}, \mathrm{q}, J_{\mathrm{C}-\mathrm{F}}=321 \mathrm{~Hz}\right)$, $118.80\left(\mathrm{C}_{4 \mathrm{a}}\right), 129.48\left(\mathrm{C}_{5}\right), 142.18\left(\mathrm{C}_{4}\right), 150.84\left(\mathrm{C}_{7}\right), 154.57\left(\mathrm{C}_{8 \mathrm{a}}\right), 159.35(\mathrm{C}=\mathrm{O}) . \mathrm{EIMS}: \mathrm{m} / \mathrm{z}$ $294.9880[\mathrm{M}+\mathrm{H}]^{+}$, (calculated for $\left[\mathrm{C}_{10} \mathrm{H}_{6} \mathrm{~F}_{3} \mathrm{O}_{5} \mathrm{~S}\right]^{+}:$294.9884).

Styrene synthesis:

Method 1: Wittig Reaction under microwave conditions.

Potassium carbonate $(0.10 \mathrm{~mol})$ and (ethyl)triphenylphosphonium iodide $(0.016 \mathrm{~mol})$ were mixed and crushed in a mortar to a fine powder and transferred into a $250 \mathrm{~mL}$ Erlenmeyer flask. Aldehyde $(0.016 \mathrm{~mol})$ was added to the powdered mixture and thoroughly mixed. The Erlenmeyer flask was covered with a watch glass and placed into a microwave oven along with a $1 \mathrm{~L}$ beaker of ice (to ensure that the reaction mixture did not overheat). The microwave was run at full power for 12 minutes, making sure to pause the heating and replace the ice provided it melted. A tiny sample of the crude reaction product was removed, diluted in a small amount of hexanes and analyzed by TLC 
to check the reaction progress. Upon completion, the crude solids were extracted with hexanes $(3 \mathrm{x}$ $20 \mathrm{~mL}$ ). The combined hexane extracts were placed in a round-bottomed flask and concentrated by rotary evaporation to obtain a yellow oil which was purified by flash chromatography on silica gel using hexanes or a mixture of hexanes/ethyl acetate as eluent.

Method 2: Knoevenagel condensation reactions:

To a solution of aldehyde $(5 \mathrm{mmol})$ and malonic acid $(20 \mathrm{mmol})$ in pyridine $(21 \mathrm{~mL})$ was added piperidine $(0.75 \mathrm{~mL}, 7.6 \mathrm{mmol})$. The mixture was heated at reflux for a period of $30 \mathrm{~min}$. under microwave irradiation. Then toluene $(40 \mathrm{~mL})$ was added to the cooled reaction mixture and the solvent volume reduced in vacuo at $30-40^{\circ} \mathrm{C}$. Additional toluene $(20 \mathrm{~mL})$ was then added and the solvent again removed in vacuo in order to remove all traces of pyridine. This procedure afforded a residue which was purified by column chromatography over silica gel. Elution with 9:1 hexane/ethyl acetate afforded the corresponding vinylphenol.

General procedure for the Mizoroki-Heck reactions: Styrene (3mmol), 3-bromo-7alkyloxycoumarin (4) or 2-oxo-2H-chromen-7-yl trifluoromethanesulfonate (9) (3 mmol), triethanolamine $(3 \mathrm{~mL})$ and $\mathrm{Pd}(\mathrm{OAc})_{2}(0.03 \mathrm{~g})$ were placed in a $25 \mathrm{~mL}$ flat-bottomed flask equipped with a magnetic stirring bar. The mixture was stirred and heated to reflux for a period of $30 \mathrm{~min}$, under microwave irradiation. The mixture was allowed to cool and then passed through a pad of silica using ethyl acetate as the solvent. Then the filtrate was evaporated and the crude residue was purified by preparative chromatography (silica gel, hexane/methylene chloride (1:1) mixture) affording the coupling products in $35-58 \%$ yield.

3-[(E)-2-(2,4-dimethoxyphenyl)ethenyl]-4-methyl-7-(octyloxy)-2H-chromen-2-one

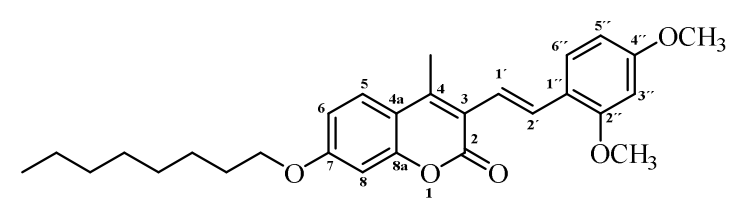

Yield: $0.39 \mathrm{mmol}, 175 \mathrm{mg}, 39 \%$; M.p. $106-108{ }^{\circ} \mathrm{C}$; IR $\left(\mathrm{KBr}, \mathrm{cm}^{-1}\right): v_{\max } 2922(\mathrm{C}-\mathrm{H}), 1714(\mathrm{C}=\mathrm{O})$, $1610(\mathrm{C}=\mathrm{C}), 1504\left(\mathrm{C}=\mathrm{C}_{\mathrm{Ar}}\right), 1251(\mathrm{C}-\mathrm{O}-\mathrm{C}), 1153((\mathrm{C}=\mathrm{O})-\mathrm{O}), 834\left(\mathrm{C}-\mathrm{H}_{\mathrm{Ar}}\right) ;{ }^{1} \mathrm{H}$ NMR $\left(\mathrm{CDCl}_{3}, 300\right.$ $\mathrm{MHz}): \delta 0.94\left(\mathrm{CH}_{3}, \mathrm{t}, J=6.8 \mathrm{~Hz}\right), 1.35\left(3 \mathrm{CH}_{2}, \mathrm{~m}\right), 1.52\left(\mathrm{CH}_{2}, \mathrm{~m}\right), 1.62\left(\mathrm{CH}_{2}, \mathrm{~m}\right), 1.86\left(\mathrm{CH}_{2}, \mathrm{~m}\right)$, $2.60\left(\mathrm{CH}_{3}, \mathrm{~s}\right), 3.89\left(\mathrm{OCH}_{3}, \mathrm{~s}\right), 3.91\left(\mathrm{OCH}_{3}, \mathrm{~s}\right) 4.06\left(-\mathrm{CH}_{2} \mathrm{O}-, \mathrm{t}, J=6.5 \mathrm{~Hz}\right), 6.51\left(\mathrm{H}_{3^{\prime \prime}}, \mathrm{d}, J=2.5\right.$ $\mathrm{Hz}), 6.57\left(\mathrm{H}_{5}{ }^{\prime \prime}, \mathrm{dd}, \mathrm{J}=8.8,2.5 \mathrm{~Hz}\right), 6.85\left(\mathrm{H}_{8}, \mathrm{~d}, J=2.5 \mathrm{~Hz}\right), 6.90\left(\mathrm{H}_{6}, \mathrm{dd}, J=8.8,2.5 \mathrm{~Hz}\right), 7.12$ $\left(\mathrm{H}_{1^{\prime}}, \mathrm{d}, J=16.6 \mathrm{~Hz}\right), 7.56\left(\mathrm{H}_{6^{\prime \prime}}, \mathrm{d}, J=8.8 \mathrm{~Hz}\right), 7.60\left(\mathrm{H}_{5}, \mathrm{~d}, \mathrm{~J}=8.8 \mathrm{~Hz}\right), 7.74\left(\mathrm{H}_{2}{ }^{\prime}, \mathrm{d}, J=16.6 \mathrm{~Hz}\right)$. ${ }^{13} \mathrm{C} \mathrm{NMR}\left(\mathrm{CDCl}_{3}, 75 \mathrm{MHz}\right): \delta 14.14\left(\mathrm{CH}_{3}\right), 15.63\left(\mathrm{CH}_{3}\right) 22.68\left(\mathrm{CH}_{2}\right), 26.0\left(\mathrm{CH}_{2}\right), 29.05\left(\mathrm{CH}_{2}\right)$, 
$29.25\left(\mathrm{CH}_{2}\right), 29.35\left(\mathrm{CH}_{2}\right), 31.82\left(\mathrm{CH}_{2}\right) 53.43\left(\mathrm{OCH}_{3}\right) 55.59\left(\mathrm{OCH}_{3}\right) 68.61\left(-\mathrm{OCH}_{2}\right), 98.46\left(\mathrm{C}_{3^{\prime \prime}}\right)$ $100.90\left(\mathrm{C}_{8}\right), 104.93\left(\mathrm{C}_{5^{\prime \prime}}\right), 112.81\left(\mathrm{C}_{6}\right), 114.45\left(\mathrm{C}_{4 \mathrm{a}}\right), 119.76\left(\mathrm{C}_{1^{\prime \prime}}\right) 119.92\left(\mathrm{C}_{1^{\prime}}\right), 120.24\left(\mathrm{C}_{3}\right) 125.82$ (C5) $127.90\left(\mathrm{C}_{6^{\prime \prime}}\right) 130.80\left(\mathrm{C}_{2}\right) 145.38\left(\mathrm{C}_{8 \mathrm{a}}\right) 153.55\left(\mathrm{C}_{4}\right), 158.39\left(\mathrm{C}_{2^{\prime \prime}}\right), 160.76\left(\mathrm{C}_{7}\right), 160.87\left(\mathrm{C}_{4^{\prime \prime}}\right)$ $161.44(\mathrm{C}=\mathrm{O})$. EIMS: $\mathrm{m} / \mathrm{z} 451.2482[\mathrm{M}+\mathrm{H}]^{+}$, (calculated for $\left[\mathrm{C}_{28} \mathrm{H}_{35} \mathrm{O}_{5}\right]^{+}:$451.2484).

3-[(E)-2-(4-methoxyphenyl)ethenyl]-4-methyl-7-(octyloxy)-2H-chromen-2-one

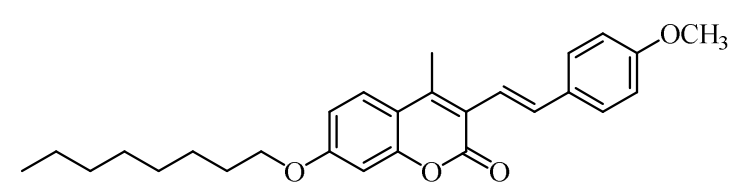

Yield: 0.37mmol, 155mg, 37\%; M.p. $114-116{ }^{\circ} \mathrm{C}$; IR $\left(\mathrm{KBr}, \mathrm{cm}^{-1}\right): v \max 2926(\mathrm{C}-\mathrm{H}), 1710(\mathrm{C}=\mathrm{O})$, $1610(\mathrm{C}=\mathrm{C}), 1512\left(\mathrm{C}=\mathrm{C}_{\mathrm{Ar}}\right), 1242(\mathrm{C}-\mathrm{O}-\mathrm{C}), 1178((\mathrm{C}=\mathrm{O})-\mathrm{O}), 834\left(\mathrm{C}-\mathrm{H}_{\mathrm{Ar}}\right) ;{ }^{1} \mathrm{H}$ NMR $\left(\mathrm{CDCl}_{3}, 300\right.$ $\mathrm{MHz}): \delta 0.94\left(\mathrm{CH}_{3}, \mathrm{t}, J=6.7 \mathrm{~Hz}\right), 1.35\left(3 \mathrm{CH}_{2}, \mathrm{~m}\right), 1.52\left(\mathrm{CH}_{2}, \mathrm{~m}\right), 1.63\left(\mathrm{CH}_{2}, \mathrm{~m}\right), 1.86\left(\mathrm{CH}_{2}, \mathrm{~m}\right)$, $2.60\left(\mathrm{CH}_{3}, \mathrm{~s}\right), 3.88\left(\mathrm{OCH}_{3}, \mathrm{~s}\right), 4.06\left(-\mathrm{CH}_{2} \mathrm{O}-, \mathrm{t}, J=6.5 \mathrm{~Hz}\right), 6.85\left(\mathrm{H}_{8}, \mathrm{~d}, J=2.5 \mathrm{~Hz}\right), 6.91\left(\mathrm{H}_{6}, \mathrm{dd}, J\right.$ = 9.0, $2.5 \mathrm{~Hz}), 6.95\left(\mathrm{H}_{3^{\prime \prime}}, \mathrm{H}_{5^{\prime \prime}}, \mathrm{d}, J=9.0 \mathrm{~Hz}\right), 7.07\left(\mathrm{H}_{1^{\prime}}, \mathrm{d}, J=16.2 \mathrm{~Hz}\right), 7.53\left(\mathrm{H}_{2^{\prime \prime}}, \mathrm{H}_{6^{\prime \prime}}, \mathrm{d}, J=9.0\right.$ $\mathrm{Hz}), 7.61\left(\mathrm{H}_{2}, \mathrm{~d}, J=16.2 \mathrm{~Hz}\right), 7.62\left(\mathrm{H}_{5}, \mathrm{~d}, \mathrm{~J}=9.0 \mathrm{~Hz}\right) .{ }^{13} \mathrm{C} \mathrm{NMR}\left(\mathrm{CDCl}_{3}, 75 \mathrm{MHz}\right): \delta 14.13\left(\mathrm{CH}_{3}\right)$, $15.47\left(\mathrm{CH}_{3}\right) 22.68\left(\mathrm{CH}_{2}\right), 25.98\left(\mathrm{CH}_{2}\right), 29.03\left(\mathrm{CH}_{2}\right), 29.23\left(\mathrm{CH}_{2}\right), 29.34\left(\mathrm{CH}_{2}\right), 31.81\left(\mathrm{CH}_{2}\right), 66.73$ $\left(\mathrm{OCH}_{3}\right), 68.59\left(-\mathrm{OCH}_{2}{ }^{-}\right), 100.93\left(\mathrm{C}_{8}\right), 112.90\left(\mathrm{C}_{6}\right), 114.11\left(\mathrm{C}_{3^{\prime \prime}}, \mathrm{C}_{5^{\prime \prime}}\right), 114.34\left(\mathrm{C}_{4 \mathrm{a}}\right), 118.89\left(\mathrm{C}_{1^{\prime}}\right)$, $119.34\left(\mathrm{C}_{3}\right), 125.86\left(\mathrm{C}_{5}\right), 128.02\left(\mathrm{C}_{2^{\prime}}, \mathrm{C}_{6^{\prime}}\right), 130.53\left(\mathrm{C}_{1^{\prime \prime}}\right), 135.08\left(\mathrm{C}_{2^{\prime}}\right), 147.75\left(\mathrm{C}_{8 \mathrm{a}}\right), 153.57\left(\mathrm{C}_{4}\right)$, $159.66\left(\mathrm{C}_{4^{\prime}}\right), 160.57\left(\mathrm{C}_{7}\right), 161.62(\mathrm{C}=\mathrm{O})$. EIMS: $\mathrm{m} / \mathrm{z} 421.2377[\mathrm{M}+\mathrm{H}]^{+}$, (calculated for $\left[\mathrm{C}_{27} \mathrm{H}_{33} \mathrm{O}_{4}\right]^{+}:$421.2379).

3-[(E)-2-(3,4-dimethoxyphenyl)ethenyl]-4-methyl-7-(octyloxy)-2H-chromen-2-one

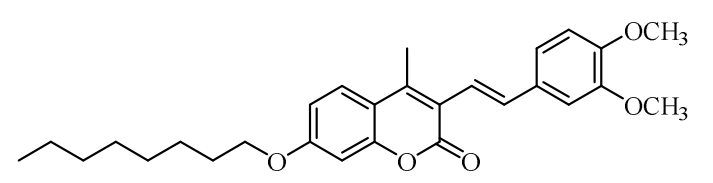

Yield: 0.38mmol, 171mg, 38\%; M.p. 94-96 ${ }^{\circ} \mathrm{C}$; IR $\left(\mathrm{KBr}, \mathrm{cm}^{-1}\right): v \max 2920(\mathrm{C}-\mathrm{H}), 1712(\mathrm{C}=\mathrm{O})$, $1618(\mathrm{C}=\mathrm{C}), 1514\left(\mathrm{C}=\mathrm{C}_{\mathrm{Ar}}\right), 1251(\mathrm{C}-\mathrm{O}-\mathrm{C}), 1161((\mathrm{C}=\mathrm{O})-\mathrm{O}), 813\left(\mathrm{C}-\mathrm{H}_{\mathrm{Ar}}\right) ;{ }^{1} \mathrm{H}$ NMR $\left(\mathrm{CDCl}_{3}, 300\right.$ $\mathrm{MHz}): \delta 0.94\left(\mathrm{CH}_{3}, \mathrm{t}, J=6.8 \mathrm{~Hz}\right), 1.35\left(3 \mathrm{CH}_{2}, \mathrm{~m}\right), 1.52\left(\mathrm{CH}_{2}, \mathrm{~m}\right), 1.62\left(\mathrm{CH}_{2}, \mathrm{~m}\right), 1.87\left(\mathrm{CH}_{2}, \mathrm{~m}\right)$, $2.61\left(\mathrm{CH}_{3}, \mathrm{~s}\right), 3.96\left(\mathrm{OCH}_{3}, \mathrm{~s}\right), 3.99\left(\mathrm{OCH}_{3}, \mathrm{~s}\right), 4.07\left(-\mathrm{CH}_{2} \mathrm{O}-, \mathrm{t}, J=6.5 \mathrm{~Hz}\right), 6.86\left(\mathrm{H}_{8}, \mathrm{~d}, J=2.5 \mathrm{~Hz}\right)$, $6.92\left(\mathrm{H}_{6}, \mathrm{H}_{5}{ }^{\prime \prime}, \mathrm{d}, J=8.8,2.5 \mathrm{~Hz}\right), 7.07\left(\mathrm{H}_{1^{\prime}}, \mathrm{d}, J=16.6 \mathrm{~Hz}\right), 7.13\left(\mathrm{H}_{2}{ }^{\prime \prime}, \mathrm{d}, J=1.8 \mathrm{~Hz}\right), 7.15\left(\mathrm{H}_{6 \prime \prime}\right.$, $\mathrm{dd}, J=8.8,1.8 \mathrm{~Hz}), 7.57\left(\mathrm{H}_{2}, \mathrm{~d}, J=16.6 \mathrm{~Hz}\right), 7.62\left(\mathrm{H}_{5}, \mathrm{~d}, \mathrm{~J}=8.8 \mathrm{~Hz}\right) .{ }^{13} \mathrm{C} \mathrm{NMR}\left(\mathrm{CDCl}_{3}, 75\right.$ $\mathrm{MHz}): \delta 14.12\left(\mathrm{CH}_{3}\right), 15.54\left(\mathrm{CH}_{3}\right), 22.68\left(\mathrm{CH}_{2}\right), 26.02\left(\mathrm{CH}_{2}\right), 29.03\left(\mathrm{CH}_{2}\right), 29.24\left(\mathrm{CH}_{2}\right), 29.35$ $\left(\mathrm{CH}_{2}\right), 31.81\left(\mathrm{CH}_{2}\right), 53.98\left(2 \mathrm{OCH}_{3}\right), 68.71\left(-\mathrm{OCH}_{2}\right), 100.90\left(\mathrm{C}_{8}\right), 109.37\left(\mathrm{C}_{2^{\prime \prime}}\right), 111.21\left(\mathrm{C}_{6}\right)$, $112.98\left(\mathrm{C}_{5^{\prime \prime}}\right), 114.31\left(\mathrm{C}_{4 \mathrm{a}}\right), 119.17\left(\mathrm{C}_{6^{\prime \prime}}\right), 119.30\left(\mathrm{C}_{3}\right), 119.90\left(\mathrm{C}_{1^{\prime}}\right), 125.87\left(\mathrm{C}_{5}\right), 130.86\left(\mathrm{C}_{1^{\prime \prime}}\right)$, 
$135.37\left(\mathrm{C}_{2}{ }^{\prime}\right), 145.90\left(\mathrm{C}_{3^{\prime \prime}}\right), 149.11\left(\mathrm{C}_{4^{\prime \prime}}\right), 149.27\left(\mathrm{C}_{8 \mathrm{a}}\right) 153.59\left(\mathrm{C}_{4}\right), 160.61\left(\mathrm{C}_{7}\right), 161.65(\mathrm{C}=\mathrm{O})$. EIMS: $\mathrm{m} / \mathrm{z} 451.2483[\mathrm{M}+\mathrm{H}]^{+}$, (calculated for $\left.\left[\mathrm{C}_{28} \mathrm{H}_{35} \mathrm{O}_{5}\right]^{+}: 451.2484\right)$.

3-[(E)-2-(4-hydroxy-3-methoxyphenyl)ethenyl]-4-methyl-7-(octyloxy)-2H-chromen-2-one

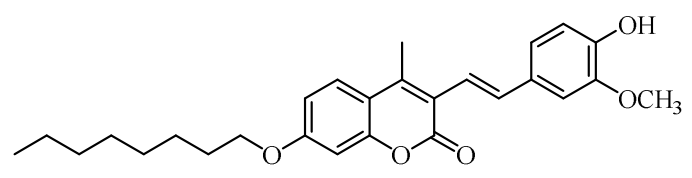

Yield: 0.36mmol, 157mg, 36\%; M.p. $114-116{ }^{\circ} \mathrm{C}$; IR $\left(\mathrm{KBr}, \mathrm{cm}^{-1}\right): v \max 2924(\mathrm{C}-\mathrm{H}), 1716(\mathrm{C}=\mathrm{O})$, $1610(\mathrm{C}=\mathrm{C}), 1512\left(\mathrm{C}=\mathrm{C}_{\mathrm{Ar}}\right), 1257(\mathrm{C}-\mathrm{O}-\mathrm{C}), 1161((\mathrm{C}=\mathrm{O})-\mathrm{O}), 813\left(\mathrm{C}-\mathrm{H}_{\mathrm{Ar}}\right) ;{ }^{1} \mathrm{H}$ NMR $\left(\mathrm{CDCl}_{3}, 300\right.$ $\mathrm{MHz}): \delta 0.94\left(\mathrm{CH}_{3}, \mathrm{t}, J=6.8 \mathrm{~Hz}\right), 1.34\left(3 \mathrm{CH}_{2}, \mathrm{~m}\right), 1.52\left(\mathrm{CH}_{2}, \mathrm{~m}\right), 1.69\left(\mathrm{CH}_{2}, \mathrm{~m}\right), 1.86\left(\mathrm{CH}_{2}, \mathrm{~m}\right)$, $2.60\left(\mathrm{CH}_{3}, \mathrm{~s}\right), 3.99\left(\mathrm{OCH}_{3}, \mathrm{~s}\right), 4.06\left(-\mathrm{CH}_{2} \mathrm{O}-, \mathrm{t}, J=6.5 \mathrm{~Hz}\right), 6.85\left(\mathrm{H}_{8}, \mathrm{~d}, J=2.5 \mathrm{~Hz}\right), 6.91\left(\mathrm{H}_{6}, \mathrm{dd}, J\right.$ $=8.8,2.5 \mathrm{~Hz}), 6.96\left(\mathrm{H}_{5^{\prime \prime}}, \mathrm{d}, J=8.8 \mathrm{~Hz}\right), 7.04\left(\mathrm{H}_{1^{\prime}}, \mathrm{d}, J=16.3 \mathrm{~Hz}\right), 7.10\left(\mathrm{H}_{2^{\prime \prime}}, \mathrm{d}, J=2.5 \mathrm{~Hz}\right), 7.12$ $\left(\mathrm{H}_{6^{\prime \prime}}, \mathrm{dd}, J=8.8,2.5 \mathrm{~Hz}\right), 7.55\left(\mathrm{H}_{2^{\prime}}, \mathrm{d}, J=16.3 \mathrm{~Hz}\right), 7.62\left(\mathrm{H}_{5}, \mathrm{~d}, \mathrm{~J}=8.8 \mathrm{~Hz}\right) .{ }^{13} \mathrm{C} \mathrm{NMR}\left(\mathrm{CDCl}_{3}, 75\right.$ $\mathrm{MHz}): \delta 14.12\left(\mathrm{CH}_{3}\right), 15.52\left(\mathrm{CH}_{3}\right), 22.66\left(\mathrm{CH}_{2}\right), 25.99\left(\mathrm{CH}_{2}\right), 29.03\left(\mathrm{CH}_{2}\right), 29.24\left(\mathrm{CH}_{2}\right), 29.36$ $\left(\mathrm{CH}_{2}\right), 31.83\left(\mathrm{CH}_{2}\right), 56.0\left(\mathrm{OCH}_{3}\right), 68.67\left(-\mathrm{OCH}_{2}-\right), 100.90\left(\mathrm{C}_{8}\right), 109.03\left(\mathrm{C}_{2}{ }^{\prime \prime}\right), 112.96\left(\mathrm{C}_{6}\right), 114.31$ $\left(\mathrm{C}_{4 \mathrm{a}}\right), 114.58\left(\mathrm{C}_{5^{\prime \prime}}\right), 118.85\left(\mathrm{C}_{6^{\prime \prime}}\right), 119.33\left(\mathrm{C}_{3}\right), 120.29\left(\mathrm{C}_{1^{\prime}}\right), 125.89\left(\mathrm{C}_{5}\right), 130.38\left(\mathrm{C}_{1^{\prime \prime}}\right), 135.56\left(\mathrm{C}_{2^{\prime}}\right)$, $145.83\left(\mathrm{C}_{4^{\prime \prime}}\right), 145.95\left(\mathrm{C}_{3^{\prime \prime}}\right), 146.69\left(\mathrm{C}_{4}\right), 153.56\left(\mathrm{C}_{8 \mathrm{a}}\right) 160.64\left(\mathrm{C}_{7}\right), 161.62(\mathrm{C}=\mathrm{O}) . \mathrm{EIMS}: \mathrm{m} / \mathrm{z}$ 437.2331 $[\mathrm{M}+\mathrm{H}]^{+}$, (calculated for $\left[\mathrm{C}_{27} \mathrm{H}_{33} \mathrm{O}_{5}\right]^{+}:$437.2328).

3-[(E)-2-(4-hydroxy-3,5-dimethoxyphenyl)ethenyl]-4-methyl-7-(octyloxy)-2H-chromen-2-one

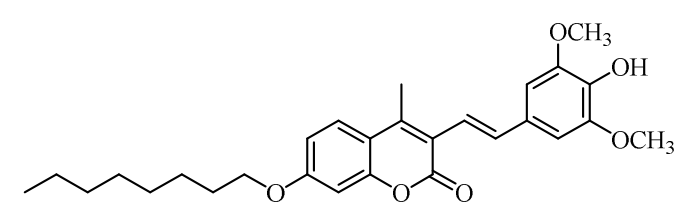

Yield: $0.41 \mathrm{mmol}, 191 \mathrm{mg}, 41 \%$; M.p. $55-57{ }^{\circ} \mathrm{C}$; IR $\left(\mathrm{KBr}, \mathrm{cm}^{-1}\right): v \max 2927(\mathrm{C}-\mathrm{H}), 1708(\mathrm{C}=\mathrm{O})$, $1616(\mathrm{C}=\mathrm{C}), 1514\left(\mathrm{C}=\mathrm{C}_{\mathrm{Ar}}\right), 1253(\mathrm{C}-\mathrm{O}-\mathrm{C}), 1153((\mathrm{C}=\mathrm{O})-\mathrm{O}), 858\left(\mathrm{C}-\mathrm{H}_{\mathrm{Ar}}\right) ;{ }^{1} \mathrm{H}$ NMR $\left(\mathrm{CDCl}_{3}, 300\right.$ $\mathrm{MHz}): \delta 0.94\left(\mathrm{CH}_{3}, \mathrm{t}, J=6.8 \mathrm{~Hz}\right), 1.34\left(3 \mathrm{CH}_{2}, \mathrm{~m}\right), 1.52\left(\mathrm{CH}_{2}, \mathrm{~m}\right), 1.66\left(\mathrm{CH}_{2}, \mathrm{~m}\right), 1.86\left(\mathrm{CH}_{2}, \mathrm{~m}\right)$, $2.61\left(\mathrm{CH}_{3}, \mathrm{~s}\right), 3.99\left(2 \mathrm{OCH}_{3}, \mathrm{~s}\right), 4.02\left(-\mathrm{CH}_{2} \mathrm{O}-, \mathrm{t}, J=6.5 \mathrm{~Hz}\right), 6.82\left(\mathrm{H}_{2}{ }^{\prime \prime}, \mathrm{H}_{6}{ }^{\prime \prime}, \mathrm{s}\right), 6.85\left(\mathrm{H}_{8}, \mathrm{~d}, J=2.0\right.$ $\mathrm{Hz}), 6.91\left(\mathrm{H}_{6}, \mathrm{dd}, J=8.9,2.0 \mathrm{~Hz}\right), 7.04\left(\mathrm{H}_{1^{\prime}}, \mathrm{d}, J=16.1 \mathrm{~Hz}\right), 7.54\left(\mathrm{H}_{2^{\prime}}, \mathrm{d}, J=16.1 \mathrm{~Hz}\right), 7.62\left(\mathrm{H}_{5}, \mathrm{~d}\right.$, $\mathrm{J}=8.9 \mathrm{~Hz}) .{ }^{13} \mathrm{C} \mathrm{NMR}\left(\mathrm{CDCl}_{3}, 75 \mathrm{MHz}\right): \delta 14.12\left(\mathrm{CH}_{3}\right), 15.58\left(\mathrm{CH}_{3}\right), 22.67\left(\mathrm{CH}_{2}\right), 26.0\left(\mathrm{CH}_{2}\right)$, $29.03\left(\mathrm{CH}_{2}\right), 29.24\left(\mathrm{CH}_{2}\right), 29.34\left(\mathrm{CH}_{2}\right), 31.83\left(\mathrm{CH}_{2}\right), 56.38\left(2 \mathrm{OCH}_{3}\right), 68.67\left(-\mathrm{OCH}_{2}-\right), 100.90\left(\mathrm{C}_{8}\right)$, $103.66 \quad\left(\mathrm{C}_{2^{\prime \prime}}, \mathrm{C}_{6^{\prime \prime}}\right), \quad 112.99 \quad\left(\mathrm{C}_{6}\right), \quad 114.26 \quad\left(\mathrm{C}_{4 \mathrm{a}}\right), \quad 119.10 \quad\left(\mathrm{C}_{1^{\prime}}\right), 119.18 \quad\left(\mathrm{C}_{3}\right), \quad 125.91 \quad\left(\mathrm{C}_{5}\right)$, 129.31 $\left(\mathrm{C}_{2^{\prime}}\right), 135.21\left(\mathrm{C}_{1^{\prime \prime}}\right), 135.78\left(\mathrm{C}_{4^{\prime \prime}}\right), 145.96\left(\mathrm{C}_{4}\right), 147.22\left(\mathrm{C}_{3^{\prime \prime}}, \mathrm{C}_{5^{\prime \prime}}\right), 160.61\left(\mathrm{C}_{4 \mathrm{a}}\right), 161.70\left(\mathrm{C}_{7}\right)$, 162.47 (C=O). EIMS: m/z 467.2426 [M+ H] $]^{+}$, (calculated for $\left[\mathrm{C}_{28} \mathrm{H}_{35} \mathrm{O}_{6}\right]^{+}$: 467.2434). 
3-[(E)-2-(1,3-benzodioxol-5-yl)ethenyl]-4-methyl-7-(octyloxy)-2H-chromen-2-one

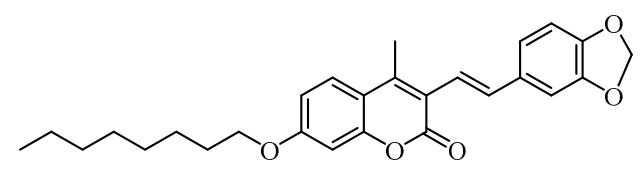

Yield: 0.49mmol, 214mg, 49\%; M.p. 122-125 ${ }^{\circ} \mathrm{C}$; IR (KBr, $\left.\mathrm{cm}^{-1}\right): v_{\max } 2926(\mathrm{C}-\mathrm{H}), 1716(\mathrm{C}=\mathrm{O})$, $1614(\mathrm{C}=\mathrm{C}), 1500\left(\mathrm{C}=\mathrm{C}_{\mathrm{Ar}}\right), 1234(\mathrm{C}-\mathrm{O}-\mathrm{C}), 1178((\mathrm{C}=\mathrm{O})-\mathrm{O}), 808\left(\mathrm{C}-\mathrm{H}_{\mathrm{Ar}}\right) ;{ }^{1} \mathrm{H}$ NMR $\left(\mathrm{CDCl}_{3}, 300\right.$ $\mathrm{MHz}): \delta 0.94\left(\mathrm{CH}_{3}, \mathrm{t}, J=6.8 \mathrm{~Hz}\right), 1.35\left(3 \mathrm{CH}_{2}, \mathrm{~m}\right), 1.51\left(\mathrm{CH}_{2}, \mathrm{~m}\right), 1.67\left(\mathrm{CH}_{2}, \mathrm{~m}\right), 1.86\left(\mathrm{CH}_{2}, \mathrm{~m}\right)$, $2.58\left(\mathrm{CH}_{3}, \mathrm{~s}\right), 4.05\left(-\mathrm{CH}_{2} \mathrm{O}-, \mathrm{t}, J=6.5 \mathrm{~Hz}\right), 6.02\left(\mathrm{O}_{-} \mathrm{CH}_{2}-\mathrm{O}\right) 6.83\left(\mathrm{H}_{8}, \mathrm{~d}, J=2.5 \mathrm{~Hz}\right), 6.84\left(\mathrm{H}_{5^{\prime \prime}}, \mathrm{d}, J\right.$ = 8.8, $2.5 \mathrm{~Hz}), 6.90\left(\mathrm{H}_{6}, \mathrm{dd}, J=8.8,2.5 \mathrm{~Hz}\right), 7.01\left(\mathrm{H}_{1^{\prime}}, \mathrm{H}_{6^{\prime \prime}}, \mathrm{m}\right), 7.12\left(\mathrm{H}_{2^{\prime \prime}}, \mathrm{d}, J=2.5 \mathrm{~Hz}\right), 7.59\left(\mathrm{H}_{2}^{\prime}\right.$, $\mathrm{d}, J=16.1 \mathrm{~Hz}), 7.60\left(\mathrm{H}_{5}, \mathrm{~d}, \mathrm{~J}=9.1 \mathrm{~Hz}\right) .{ }^{13} \mathrm{C} \mathrm{NMR}\left(\mathrm{CDCl}_{3}, 75 \mathrm{MHz}\right): \delta 14.13\left(\mathrm{CH}_{3}\right), 15.37\left(\mathrm{CH}_{3}\right)$, $22.66\left(\mathrm{CH}_{2}\right), 25.99\left(\mathrm{CH}_{2}\right), 29.03\left(\mathrm{CH}_{2}\right), 29.24\left(\mathrm{CH}_{2}\right), 29.34\left(\mathrm{CH}_{2}\right), 31.81\left(\mathrm{CH}_{2}\right), 68.60\left(-\mathrm{OCH}_{2}\right)$, $100.83\left(\mathrm{C}_{8}\right), 101.18\left(\mathrm{O}-\mathrm{CH}_{2}-\mathrm{O}\right), 105.53\left(\mathrm{C}_{2 \prime \prime}\right), 108.45\left(\mathrm{C}_{5^{\prime \prime}}\right), 112.94\left(\mathrm{C}_{6}\right), 114.20\left(\mathrm{C}_{4 \mathrm{a}}\right), 118.99$ $\left(\mathrm{C}_{6^{\prime \prime}}\right), 119.24\left(\mathrm{C}_{1^{\prime}}\right), 122.07\left(\mathrm{C}_{3}\right), 125.87\left(\mathrm{C}_{5}\right), 132.33\left(\mathrm{C}_{1^{\prime \prime}}\right), 135.07\left(\mathrm{C}_{2^{\prime}}\right), 146.02\left(\mathrm{C}_{4^{\prime \prime}}\right), 147.64$ $\left(\mathrm{C}_{3^{\prime \prime}}\right), 148.17\left(\mathrm{C}_{8 \mathrm{a}}\right) 153.57\left(\mathrm{C}_{4}\right), 160.45\left(\mathrm{C}_{7}\right), 161.66(\mathrm{C}=\mathrm{O})$. EIMS: m/z 435.2179 [M + H] $]^{+}$, (calculated for $\left[\mathrm{C}_{27} \mathrm{H}_{31} \mathrm{O}_{5}\right]^{+}:$435.2171).

3-[(E)-2-(2,5-dimethoxyphenyl)ethenyl]-4-methyl-7-(octyloxy)-2H-chromen-2-one

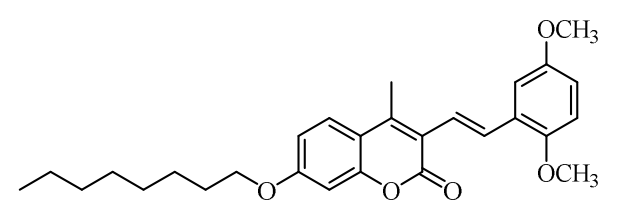

Yield: 0.35mmol, 158mg, 35\%; M.p. $74-77^{\circ} \mathrm{C}$; IR $\left(\mathrm{KBr}, \mathrm{cm}^{-1}\right): v \max 2922(\mathrm{C}-\mathrm{H}), 1705(\mathrm{C}=\mathrm{O})$, $1614(\mathrm{C}=\mathrm{C}), 1489\left(\mathrm{C}=\mathrm{C}_{\mathrm{Ar}}\right), 1253(\mathrm{C}-\mathrm{O}-\mathrm{C}), 1184((\mathrm{C}=\mathrm{O})-\mathrm{O}), 790\left(\mathrm{C}-\mathrm{H}_{\mathrm{Ar}}\right) ;{ }^{1} \mathrm{H}$ NMR $\left(\mathrm{CDCl}_{3}, 300\right.$ $\mathrm{MHz}): \delta 0.94\left(\mathrm{CH}_{3}, \mathrm{t}, J=6.9 \mathrm{~Hz}\right), 1.35\left(3 \mathrm{CH}_{2}, \mathrm{~m}\right), 1.52\left(\mathrm{CH}_{2}, \mathrm{~m}\right), 1.63\left(\mathrm{CH}_{2}, \mathrm{~m}\right), 1.86\left(\mathrm{CH}_{2}, \mathrm{~m}\right)$, $2.61\left(\mathrm{CH}_{3}, \mathrm{~s}\right), 3.87\left(\mathrm{OCH}_{3}, \mathrm{~s}\right), 3.89\left(\mathrm{OCH}_{3}, \mathrm{~s}\right) 4.06\left(-\mathrm{CH}_{2} \mathrm{O}-, \mathrm{t}, J=6.5 \mathrm{~Hz}\right), 6.86\left(\mathrm{H}_{8}, \mathrm{H}_{3^{\prime \prime}}, \mathrm{H}_{4}{ }^{\prime \prime}, \mathrm{m}\right)$, $6.91\left(\mathrm{H}_{6}, \mathrm{dd}, J=8.9,2.5 \mathrm{~Hz}\right), 7.19\left(\mathrm{H}_{6^{\prime \prime}}, \mathrm{d}, J=2.7 \mathrm{~Hz}\right), 7.22\left(\mathrm{H}_{1^{\prime}}, \mathrm{d}, J=16.1 \mathrm{~Hz}\right), 7.61\left(\mathrm{H}_{5}, \mathrm{~d}, \mathrm{~J}=\right.$ $8.9 \mathrm{~Hz}), 7.81\left(\mathrm{H}_{2}, \mathrm{~d}, J=16.1 \mathrm{~Hz}\right) .{ }^{13} \mathrm{C} \mathrm{NMR}\left(\mathrm{CDCl}_{3}, 75 \mathrm{MHz}\right): \delta 14.13\left(\mathrm{CH}_{3}\right), 15.64\left(\mathrm{CH}_{3}\right), 22.68$ $\left(\mathrm{CH}_{2}\right), 26.0\left(\mathrm{CH}_{2}\right), 29.05\left(\mathrm{CH}_{2}\right), 29.25\left(\mathrm{CH}_{2}\right), 29.35\left(\mathrm{CH}_{2}\right), 31.81\left(\mathrm{CH}_{2}\right), 55.84\left(2 \mathrm{OCH}_{3}\right), 68.61(-$ $\left.\mathrm{OCH}_{2^{-}}\right), 100.91\left(\mathrm{C}_{8}\right), 112.24\left(\mathrm{C}_{6}, \mathrm{C}_{3^{\prime \prime}}\right), 112.84\left(\mathrm{C}_{4 \mathrm{a}}\right), 114.12\left(\mathrm{C}_{4^{\prime \prime}}\right), 114.32\left(\mathrm{C}_{6^{\prime \prime}}\right), 119.80\left(\mathrm{C}_{1^{\prime}}\right), 122.17$ $\left(\mathrm{C}_{1^{\prime \prime}}\right), 126.0\left(\mathrm{C}_{3}\right), 127.65\left(\mathrm{C}_{5}\right), 130.80\left(\mathrm{C}_{2^{\prime}}\right), 146.36\left(\mathrm{C}_{2^{\prime \prime}}\right), 151.76\left(\mathrm{C}_{5^{\prime \prime}}\right), 153.71\left(\mathrm{C}_{8 \mathrm{a}}\right), 153.73\left(\mathrm{C}_{4}\right)$, $160.60\left(\mathrm{C}_{7}\right), 161.66(\mathrm{C}=\mathrm{O})$. EIMS: $\mathrm{m} / \mathrm{z} 451.2484[\mathrm{M}+\mathrm{H}]^{+},\left(\right.$calculated for $\left[\mathrm{C}_{28} \mathrm{H}_{35} \mathrm{O}_{5}\right]^{+}:$451.2484). 
7-[(E)-2-(2,5-dimethoxyphenyl)ethenyl]-2H-chromen-2-one

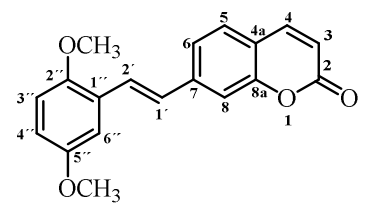

Yield: $0.51 \mathrm{mmol}, 158 \mathrm{mg}, 51 \%$; yellow solid, M.p. $125-128{ }^{\circ} \mathrm{C}$; IR $\left(\mathrm{KBr}, \mathrm{cm}^{-1}\right): v$ max $1724(\mathrm{C}=\mathrm{O})$, $1606(\mathrm{C}=\mathrm{C}), 1494\left(\mathrm{C}=\mathrm{C}_{\mathrm{Ar}}\right), 1220(\mathrm{C}-\mathrm{O}-\mathrm{C}), 1041((\mathrm{C}=\mathrm{O})-\mathrm{O}), 833\left(\mathrm{C}-\mathrm{H}_{\mathrm{Ar}}\right) ;{ }^{1} \mathrm{H}$ NMR $\left(\mathrm{CDCl}_{3}, 500\right.$ $\mathrm{MHz})$ : $\delta 3.84\left(\mathrm{OCH}_{3}, \mathrm{~s}\right), 3.89\left(\mathrm{OCH}_{3}, \mathrm{~s}\right) 6.39\left(\mathrm{H}_{3}, \mathrm{~d}, J=9.6 \mathrm{~Hz}\right), 6.84-6.88\left(\mathrm{H}_{3}{ }^{\prime \prime}, \mathrm{H}_{4}{ }^{\prime \prime}, \mathrm{m}\right), 7.12$

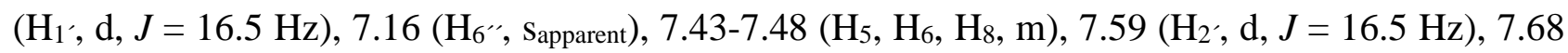
$\left(\mathrm{H}_{4}, \mathrm{~d}, J=9.6 \mathrm{~Hz}\right) .{ }^{13} \mathrm{C} \mathrm{NMR}\left(\mathrm{CDCl}_{3}, 125 \mathrm{MHz}\right): \delta 55.8\left(\mathrm{OCH}_{3}\right) 56.2\left(\mathrm{OCH}_{3}\right) 111.9\left(\mathrm{C}_{3^{\prime \prime}}\right), 112.4$ $\left(\mathrm{C}_{6^{\prime \prime}}\right), 114.3\left(\mathrm{C}_{8}\right), 114.8\left(\mathrm{C}_{4^{\prime \prime}}\right), 115.8\left(\mathrm{C}_{3}\right) 117.9\left(\mathrm{C}_{4 \mathrm{a}}\right), 122.6\left(\mathrm{C}_{6}\right) 126.2\left(\mathrm{C}_{1^{\prime \prime}}\right) 126.8\left(\mathrm{C}_{1^{\prime}}\right) 127.4\left(\mathrm{C}_{2^{\prime}}\right)$ $127.9\left(\mathrm{C}_{5}\right) 142.0\left(\mathrm{C}_{7}\right), 143\left(\mathrm{C}_{4}\right), 151.8\left(\mathrm{C}_{2^{\prime \prime}}\right), 153.8\left(\mathrm{C}_{5^{\prime \prime}}\right), 154.6\left(\mathrm{C}_{9}\right), 160.9(\mathrm{C}=\mathrm{O})$.

EIMS: m/z $309.1127[\mathrm{M}+\mathrm{H}]^{+}$, (calculated for $\left.\left[\mathrm{C}_{19} \mathrm{H}_{17} \mathrm{O}_{4}\right]^{+}: 309.1134\right)$.

7-[(E)-2-(2,3-dimethoxyphenyl)ethenyl]-2H-chromen-2-one

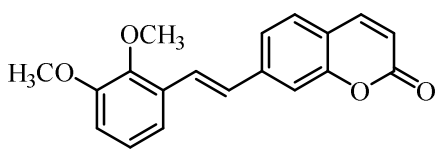

Yield: 0.58mmol, 179mg, 58\%; orange solid, M.p. $154-157^{\circ} \mathrm{C}$; IR $\left(\mathrm{KBr}, \mathrm{cm}^{-1}\right): v \max 1720(\mathrm{C}=\mathrm{O})$, $1604(\mathrm{C}=\mathrm{C}), 1475\left(\mathrm{C}=\mathrm{C}_{\mathrm{Ar}}\right), 1276(\mathrm{C}-\mathrm{O}-\mathrm{C}), 1143((\mathrm{C}=\mathrm{O})-\mathrm{O}), 837\left(\mathrm{C}-\mathrm{H}_{\mathrm{Ar}}\right) ;{ }^{1} \mathrm{H}$ NMR $\left(\mathrm{CDCl}_{3}, 500\right.$ $\mathrm{MHz}): \delta 3.91\left(\mathrm{OCH}_{3}, \mathrm{~s}\right), 3.92\left(\mathrm{OCH}_{3}, \mathrm{~s}\right) 6.41\left(\mathrm{H}_{3}, \mathrm{~d}, J=9.4 \mathrm{~Hz}\right), 6.91\left(\mathrm{H}_{4^{\prime \prime}}, \mathrm{dd}, J=8.1,1.3 \mathrm{~Hz}\right)$, $7.11\left(\mathrm{H}_{5^{\prime}}, \mathrm{dd}, J=8.0,8.0 \mathrm{~Hz}\right), 7.17\left(\mathrm{H}_{1^{\prime}}, \mathrm{d}, J=16.5 \mathrm{~Hz}\right), 7.28-7.30\left(\mathrm{H}_{6}{ }^{\prime \prime}, \mathrm{m}\right), 7.46-7.51\left(\mathrm{H}_{5}, \mathrm{H}_{6}, \mathrm{H}_{8}\right.$, m), $7.61\left(\mathrm{H}_{2}, \mathrm{~d}, J=16.4 \mathrm{~Hz}\right), 7.71\left(\mathrm{H}_{4}, \mathrm{~d}, J=9.5 \mathrm{~Hz}\right) .{ }^{13} \mathrm{C} \mathrm{NMR}\left(\mathrm{CDCl}_{3}, 125 \mathrm{MHz}\right): \delta 55.9$ $\left(\mathrm{OCH}_{3}\right), 61.1\left(\mathrm{OCH}_{3}\right) 112.3\left(\mathrm{C}_{4 \prime \prime}\right), 114.4\left(\mathrm{C}_{8}\right), 115.9\left(\mathrm{C}_{3}\right), 118.0\left(\mathrm{C}_{4 \mathrm{a}}\right), 122.6\left(\mathrm{C}_{6}\right) 124.2\left(\mathrm{C}_{5^{\prime \prime}}\right) 126.4$ $\left(\mathrm{C}_{1^{\prime}}{ }^{\prime}\right) 127.9\left(\mathrm{C}_{2^{\prime}}\right) 128.0\left(\mathrm{C}_{5}\right) 130.6\left(\mathrm{C}_{1^{\prime \prime}}\right), 142.0\left(\mathrm{C}_{7}\right), 143.0\left(\mathrm{C}_{4}\right), 147.4\left(\mathrm{C}_{2^{\prime \prime}}\right), 153.1\left(\mathrm{C}_{3^{\prime \prime}}\right), 154.6$ $\left(\mathrm{C}_{8 \mathrm{a}}\right), 160.8(\mathrm{C}=\mathrm{O})$. EIMS: $\mathrm{m} / \mathrm{z} 309.1127[\mathrm{M}+\mathrm{H}]^{+}$, (calculated for $\left[\mathrm{C}_{19} \mathrm{H}_{17} \mathrm{O}_{4}\right]^{+}:$309.1131).

7-[(E)-2-(4-hydroxy-3,5-dimethoxyphenyl)ethenyl]-2H-chromen-2-one

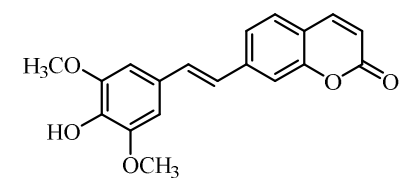

Yield: $0.51 \mathrm{mmol}, 165 \mathrm{mg}, 51 \%$; yellow solid, M.p. $159-161{ }^{\circ} \mathrm{C}$; IR $\left(\mathrm{KBr}, \mathrm{cm}^{-1}\right): v \max 3433(\mathrm{OH})$, $1724(\mathrm{C}=\mathrm{O}), 1612(\mathrm{C}=\mathrm{C}), 1512\left(\mathrm{C}=\mathrm{C}_{\mathrm{Ar}}\right), 1211(\mathrm{C}-\mathrm{O}-\mathrm{C}), 1112((\mathrm{C}=\mathrm{O})-\mathrm{O}), 833\left(\mathrm{C}-\mathrm{H}_{\mathrm{Ar}}\right) ;{ }^{1} \mathrm{H}$ NMR 
$\left(\mathrm{CDCl}_{3}, 500 \mathrm{MHz}\right): \delta 3.97\left(2-\mathrm{OCH}_{3}, \mathrm{~s}\right), 6.38\left(\mathrm{H}_{3}, \mathrm{~d}, J=9.5 \mathrm{~Hz}\right), 6.80\left(\mathrm{H}_{2}{ }^{\prime \prime}, \mathrm{H}_{6 \prime}{ }^{\prime \prime}, \mathrm{s}\right), 6.97\left(\mathrm{H}_{1^{\prime}}, \mathrm{d}, J=\right.$ 16.1 Hz), $7.15\left(\mathrm{H}_{2}, \mathrm{~d}, J=16.1 \mathrm{~Hz}\right), 7.40-7.46\left(\mathrm{H}_{5}, \mathrm{H}_{6}, \mathrm{H}_{8}, \mathrm{~m}\right), 7.68\left(\mathrm{H}_{4}, \mathrm{~d}, J=9.5 \mathrm{~Hz}\right) .{ }^{13} \mathrm{C} \mathrm{NMR}$ $\left(\mathrm{CDCl}_{3}, 125 \mathrm{MHz}\right): \delta 56.4\left(\mathrm{OCH}_{3}\right), 103.8\left(\mathrm{C}_{2}{ }^{\prime \prime}, \mathrm{C}_{6 "}{ }^{\prime \prime}\right), 103.9\left(\mathrm{C}_{8}\right), 115.2\left(\mathrm{C}_{3}\right), 117.8\left(\mathrm{C}_{4 a}\right), 122.3\left(\mathrm{C}_{6}\right)$ $125.0\left(\mathrm{C}_{1^{\prime}}\right) 127.9\left(\mathrm{C}_{5}\right) 128.0\left(\mathrm{C}_{1^{\prime \prime}}\right) 132.1\left(\mathrm{C}_{2}{ }^{\prime}\right), 135.7\left(\mathrm{C}_{4^{\prime \prime}}\right), 141.5\left(\mathrm{C}_{7}\right), 143.0\left(\mathrm{C}_{4}\right), 147.3\left(\mathrm{C}_{3^{\prime \prime}}, \mathrm{C}_{5^{\prime \prime}}\right)$, 154.6 (C9), $160.9(\mathrm{C}=\mathrm{O})$. EIMS: m/z $325.1076[\mathrm{M}+\mathrm{H}]^{+}$, (calculated for $\left.\left[\mathrm{C}_{19} \mathrm{H}_{17} \mathrm{O}_{5}\right]^{+}: 325.1069\right)$.

7-[(E)-2-(4-hydroxy-3-methoxyphenyl)ethenyl]-2H-chromen-2-one

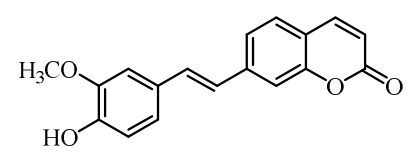

Yield: $0.50 \mathrm{mmol}, 147 \mathrm{mg}$, 52\%; yellow solid, M.p. 204-206 ${ }^{\circ} \mathrm{C}$; IR $\left(\mathrm{KBr}, \mathrm{cm}^{-1}\right): v \max 3402(\mathrm{OH})$, $1705(\mathrm{C}=\mathrm{O}), 1606(\mathrm{C}=\mathrm{C}), 1512\left(\mathrm{C}=\mathrm{C}_{\mathrm{Ar}}\right), 1267(\mathrm{C}-\mathrm{O}-\mathrm{C}), 1124((\mathrm{C}=\mathrm{O})-\mathrm{O}), 835\left(\mathrm{C}-\mathrm{H}_{\mathrm{Ar}}\right) ;{ }^{1} \mathrm{H}$ NMR $\left(\mathrm{CDCl}_{3}, 500 \mathrm{MHz}\right): \delta 3.83\left(-\mathrm{OCH}_{3}, \mathrm{~s}\right), 6.41\left(\mathrm{H}_{3}, \mathrm{~d}, J=9.5 \mathrm{~Hz}\right), 6.80\left(\mathrm{H}_{5}{ }^{\prime}, \mathrm{d}, J=8.1 \mathrm{~Hz}\right), 7.05\left(\mathrm{H}_{6}\right.$, $\mathrm{d}, J=8.3 \mathrm{~Hz}), 7.16\left(\mathrm{H}_{1^{\prime}}, \mathrm{d}, J=16.3 \mathrm{~Hz}\right), 7.25\left(\mathrm{H}_{2^{\prime \prime}}, \mathrm{s}_{\text {apparent }}\right), 7.38\left(\mathrm{H}_{2^{\prime}}, \mathrm{d}, J=16.3 \mathrm{~Hz}\right), 7.54\left(\mathrm{H}_{8}\right.$, Sapparent), $7.55\left(\mathrm{H}_{6}, \mathrm{~d}, J=8.9 \mathrm{~Hz}\right), 7.67\left(\mathrm{H}_{5}, \mathrm{~d}, J=8.0 \mathrm{~Hz}\right), 8.02\left(\mathrm{H}_{4}, \mathrm{~d}, J=9.5 \mathrm{~Hz}\right) .{ }^{13} \mathrm{C} \mathrm{NMR}$ $\left(\mathrm{CDCl}_{3}, 125 \mathrm{MHz}\right): \delta 56.1\left(\mathrm{OCH}_{3}\right), 110.7\left(\mathrm{C}_{2}^{\prime \prime}\right), 113.4\left(\mathrm{C}_{8}\right), 115.4\left(\mathrm{C}_{4 \mathrm{a}}\right), 116.5\left(\mathrm{C}_{3}\right) 117.9\left(\mathrm{C}_{5^{\prime \prime}}\right)$ $121.5\left(\mathrm{C}_{6^{\prime \prime}}\right) 122.9\left(\mathrm{C}_{6}\right) 124.2\left(\mathrm{C}_{1^{\prime}}\right), 128.4\left(\mathrm{C}_{1^{\prime \prime}}\right), 129.0\left(\mathrm{C}_{5}\right), 132.6\left(\mathrm{C}_{2^{\prime}}\right), 142.3\left(\mathrm{C}_{7}\right), 144.4\left(\mathrm{C}_{4}\right)$, $148.2\left(\mathrm{C}_{4^{\prime \prime}}\right), 148.4\left(\mathrm{C}_{3^{\prime \prime}}\right), 154.6\left(\mathrm{C}_{9}\right), 160.6(\mathrm{C}=\mathrm{O})$. EIMS: m/z $295.0970[\mathrm{M}+\mathrm{H}]^{+}$, (calculated for $^{2}$ $\left[\mathrm{C}_{18} \mathrm{H}_{15} \mathrm{O}_{4}\right]^{+}:$295.0964).

7-[(E)-2-(1,3-benzodioxol-5-yl)ethenyl]-2H-chromen-2-one

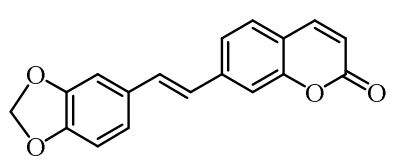

Yield: 0.55mmol, 161mg, 55\%; yellow solid, M.p. 193-196 ${ }^{\circ} \mathrm{C}$; IR $\left(\mathrm{KBr}, \mathrm{cm}^{-1}\right): v \max 1720(\mathrm{C}=\mathrm{O})$, $1606(\mathrm{C}=\mathrm{C}), 1494\left(\mathrm{C}=\mathrm{C}_{\mathrm{Ar}}\right), 1249(\mathrm{C}-\mathrm{O}-\mathrm{C}), 1041((\mathrm{C}=\mathrm{O})-\mathrm{O}), 846\left(\mathrm{C}-\mathrm{H}_{\mathrm{Ar}}\right) ;{ }^{1} \mathrm{H}$ NMR $\left(\mathrm{CDCl}_{3}, 500\right.$ MHz): $\delta 6.01\left(\mathrm{H}_{6^{\prime \prime}}, \mathrm{s}\right), 6.38\left(\mathrm{H}_{3}, \mathrm{~d}, J=9.5 \mathrm{~Hz}\right), 6.83\left(\mathrm{H}_{5^{\prime \prime}}, \mathrm{d}, J=8.0 \mathrm{~Hz}\right), 6.95\left(\mathrm{H}_{1^{\prime}}, \mathrm{d}, J=16.3 \mathrm{~Hz}\right)$, $6.99\left(\mathrm{H}_{6^{\prime \prime}}, \mathrm{dd}, J=8.0,1.5 \mathrm{~Hz}\right), 7.10\left(\mathrm{H}_{2^{\prime \prime}}, \mathrm{d}, J=1.5 \mathrm{~Hz}\right), 7.15\left(\mathrm{H}_{2}, \mathrm{~d}, J=16.3 \mathrm{~Hz}\right), 7.39\left(\mathrm{H}_{8}, \mathrm{~d}, J=\right.$ $1.5 \mathrm{~Hz}), 7.40\left(\mathrm{H}_{6}, \mathrm{~d}, J=6.0 \mathrm{~Hz}\right), 7.44\left(\mathrm{H}_{5}, \mathrm{~d}, \mathrm{~J}=8.5 \mathrm{~Hz}\right), 7.68\left(\mathrm{H}_{4}, \mathrm{~d}, J=9.6 \mathrm{~Hz}\right) .{ }^{13} \mathrm{C} \mathrm{NMR}$ $\left(\mathrm{CDCl}_{3}, 125 \mathrm{MHz}\right): \delta 101.3\left(\mathrm{OCH}_{2} \mathrm{O}\right), 105.7\left(\mathrm{C}_{2^{\prime \prime}}\right), 108.5\left(\mathrm{C}_{5^{\prime \prime}}\right), 113.9\left(\mathrm{C}_{8}\right), 115.8\left(\mathrm{C}_{4}\right), 117.9\left(\mathrm{C}_{4 \mathrm{a}}\right)$ $122.3\left(\mathrm{C}_{6^{\prime \prime}}\right) 122.4\left(\mathrm{C}_{6}\right) 125.1\left(\mathrm{C}_{5}\right), 127.9\left(\mathrm{C}_{1^{\prime}}\right), 131.0\left(\mathrm{C}_{1^{\prime \prime}}\right), 131.7\left(\mathrm{C}_{2^{\prime}}\right), 141.6\left(\mathrm{C}_{7}\right), 143.0\left(\mathrm{C}_{4}\right)$, $148.1\left(\mathrm{C}_{4^{\prime \prime}}\right), 148.3\left(\mathrm{C}_{3^{\prime \prime}}\right), 154.6\left(\mathrm{C}_{9}\right), 160.8(\mathrm{C}=\mathrm{O})$. EIMS: m/z 293.0814 [M + H $]^{+}$, (calculated for $^{\prime}$ $\left[\mathrm{C}_{18} \mathrm{H}_{13} \mathrm{O}_{4}\right]^{+}$: 293.0808). 


\section{Biological activity assays}

\section{Cell lines and culture medium}

An adenocarcinoma colon cancer cell line (SW480) and non-malignant cells (CHO-K1) were used for biological assays. These were obtained from The European Collection of Authenticated Cell Cultures (ECACC, England) and maintained in Dulbecco's Modified Eagle Medium, supplemented with $10 \%$ heat-inactivated $\left(56^{\circ} \mathrm{C}\right)$ horse serum, $1 \%$ penicillin/streptomycin and $1 \%$ non-essential amino acids (Gibco Invitrogen, Carlsbad, USA). For all experiments, horse serum was reduced to $3 \%$, and the medium was supplemented with $5 \mathrm{mg} / \mathrm{ml}$ transferrin, $5 \mathrm{ng} / \mathrm{ml}$ selenium and $10 \mathrm{mg} / \mathrm{ml}$ insulin (ITS-defined medium; Gibco, Invitrogen, Carlsbad, USA) (Maldonado et al., 2009).

\section{Cell Viability by Trypan Blue Dye Exclusion Assay}

To assess the effect of styrylcoumarins on the viability of both cell lines, a trypan blue dye exclusion test was performed, which allows to differentiate live cells since these can exclude the dye (Lamy et al., 2007). Cells were seeded to a final density of $1 \times 10^{5}$ cells/well in 12-well tissue culture plates and incubated at $37{ }^{\circ} \mathrm{C}$ in a $5 \% \mathrm{CO}_{2}$ atmosphere. The cultures were allowed to grow for $24 \mathrm{~h}$ and then were treated for 24 and $48 \mathrm{~h}$ with either $0,5 \%$ DMSO (vehicle control) or various concentrations $(10-100 \mu \mathrm{M})$ of different styrylcoumarins, as well as coumarin, resveratrol (lead compounds) and 5-fluorouracil (5-FU; the standard drug). After treatment, cells were washed with buffer versene and they were collected by trypsinization $(0.5 \%$ trypsin/2.6 $\mathrm{mM}$ ethylenediaminetetraacetic acid) followed by centrifugation. Then, trypan blue (1/1, vol/vol) was added to the pellet. The number of unstained cells (live cells) was determined by optical microscope, using hemocytometer. The inhibition percentage was calculated with regard to untreated cells (vehicle control) and $\mathrm{IC}_{50}$ values were obtained from dose response curves for each compound. Selectivity index (SI) was calculated by the ratio of $\mathrm{IC}_{50}$ values in non-malignant $\mathrm{CHO}-$ $\mathrm{K} 1$ cells to $\mathrm{IC}_{50}$ of SW480 cells.

\section{Antiproliferative activity of styrylcoumarins}

The antiproliferative effect of styrylcoumarins was evaluated by using Sulforhodamine B (SRB) dye, a colorimetric assay based on staining of total cellular protein of adherent cells. Cells were seeded to a final density of 2500 cells/well in 96-well tissue culture plates and incubated at $37^{\circ} \mathrm{C}$ in a $5 \%$ $\mathrm{CO}_{2}$ atmosphere. The cultures were allowed to grow for $24 \mathrm{~h}$ and then were treated either with increasing concentration of styrylcoumarins $(0.02-50 \mu \mathrm{M}$, the ranges depended on the result of the 
$\mathrm{IC}_{50}$ value) or $0.5 \%$ DMSO (control), for $0,2,4,6$ and 8 days. Culture media was replaced every 48 hours. After each incubation time, cells were fixed with trichloroacetic acid $(50 \% \mathrm{v} / \mathrm{v})$ (MERCK) for an hour. Cell proteins were determined by staining with $0.4 \%$ (w/v) SRB (Sigma-Aldrich, United States). Cells were then washed with $1 \%$ acetic acid for the removal of unbound SRB, and left for air-drying. Protein bound SRB was solubilized in $10 \mathrm{mM}$ Tris-base and the absorbance was measured at $492 \mathrm{~nm}$ in a microplate reader (Mindray MR-96A) (Pérez et al., 2014). All experiments were performed in triplicate.

\section{Statistical analysis}

All experiments were performed at least three times. Data are reported as mean \pm SE (standard error). Statistical differences between control group (non-treated) and treated cells were evaluated by one-way ANOVA followed by the Dunnett's test. Values with $p \leq 0.05$ were considered significant. Data were analyzed with GraphPad Prism version 7.03 for Windows (Graph Pad Software, San Diego, California, USA).

\section{Results and Discussion}

Chemistry

\section{Synthesis of 3-styrylcoumarins}

Synthesis of 3-styrylcoumarins was performed following the scheme 1, which began with formation of the coumarin (2) by microwave assisted Pechmann reaction between resorcinol (1) and ethyl acetoacetate (Manhas et al., 2006). Compound (3), obtained by microwave -assisted Williamson etherification reaction of coumarin (2) (Otero et al., 2014) in $85 \%$ yield, was brominated with NBS (Adhikari et al., 2002) to give the alkyloxybromocoumarin (4) in 75\% yield. This compound was subjected to cross-coupling with various styrenes (5) under palladium catalysis (Heck reaction) (Sabrina et al., 2011), leading to the formation of seven 3-styrylcoumarins (6a-6g) in 35\%-49\% yields. In order to improve these results other methodologies were tested such as $\left(\mathrm{Pd}\left(\mathrm{Ph}_{3}\right)_{4}, \mathrm{AgOAc}\right.$, DMF) (Martins et al., 2012) or $\mathrm{Pd}(\mathrm{AcO})_{2}, \mathrm{PPh}_{3}, \mathrm{CH}_{3} \mathrm{CO}_{2} \mathrm{Na}$ (Gordo et al., 2011) but the results were not as expected. Styrenes (5) were synthesized via microwave assisted Wittig reaction between substituted aldehydes and methyltriphenylphosphonium bromide (Martin et al., 2007) and Knoevenagel condensation reactions between 4-hydroxybenzaldehydes with malonic acid (Simpson et al., 2005). 

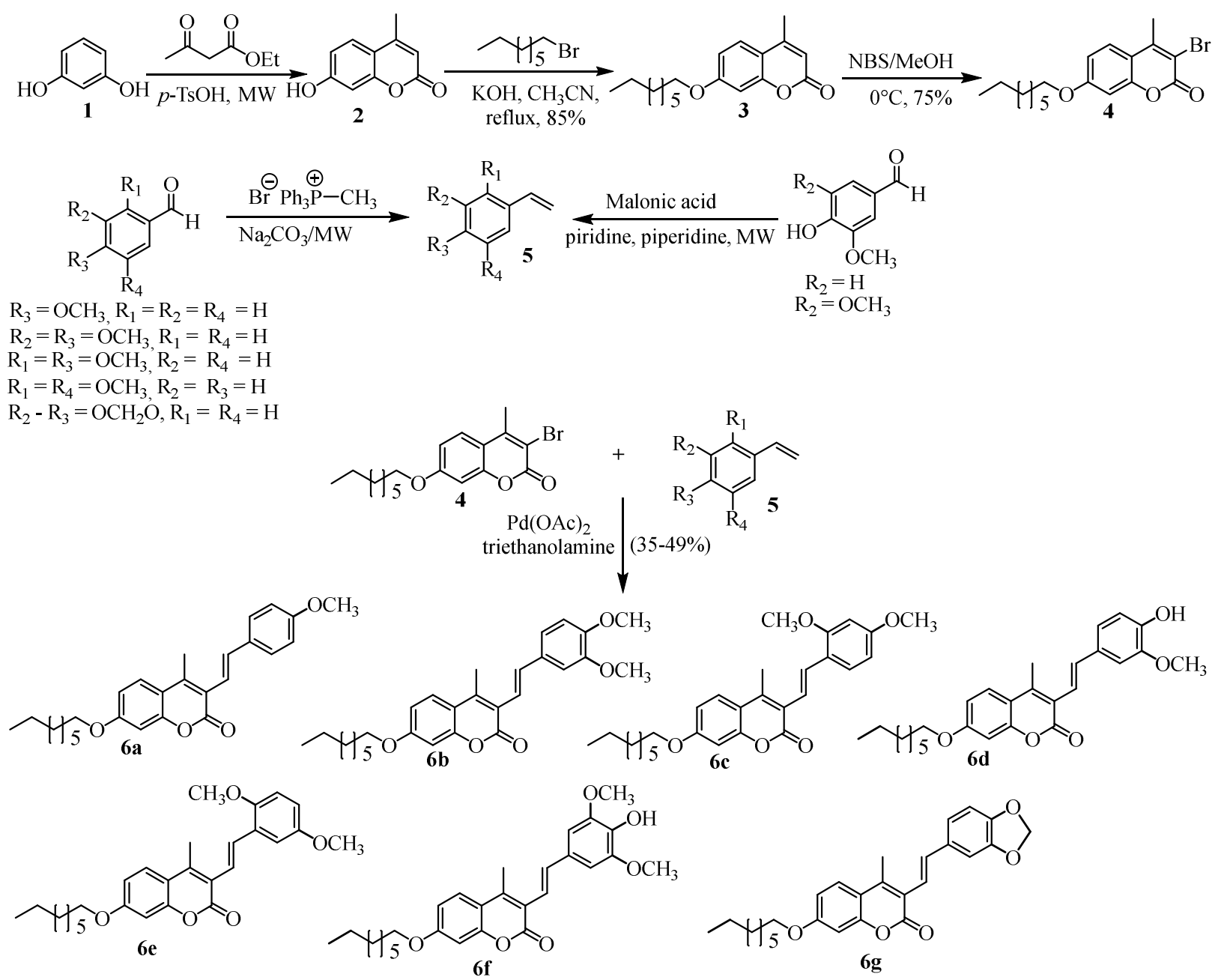

Scheme 1. Synthetic pathway to 3-styrylcoumarins

\section{Synthesis of 7-styrylcoumarins}

Scheme 2 shows the synthesis of 7-styrylcoumarins, which began with the formation of the 7hydroxycoumarin (8) by microwave assisted condensation between resorcinol (1) and malic acid (7) (Symeonidis et al., 2009). Compound (8) was treated with trifluoromethanesulfonic anhydride to obtain triflate 9 (Cano et al., 2013) in 85\% yield, that in turn was subjected to cross-coupling with various styrenes (5) under palladium catalysis (Sabrina et al., 2011), to afford the styrylcoumarins under study (10a-10e) in 50\%-58\% yields. The product of the reaction of coumarin $\mathbf{8}$ with styrenes $\mathbf{5 f}$ and $\mathbf{5 g}$ showed in the NMR duplication of several signals, which could indicate a mixture of isomers. For this reason, these compounds were not taken into account in this study. 
<smiles>O=C(O)CC(O)C(=O)O</smiles><smiles></smiles><smiles>[R3]c1cc(C=C)c([R3])c([R3])c1[R1]</smiles>

5

$$
\begin{aligned}
& \text { a. } \mathrm{R}_{1}=\mathrm{R}_{4}=\mathrm{OCH}_{3}, \mathrm{R}_{2}=\mathrm{R}_{3}=\mathrm{H} \\
& \text { b. } \mathrm{R}_{1}=\mathrm{R}_{2}=\mathrm{OCH}_{3} \mathrm{R}_{3}=\mathrm{R}_{4}=\mathrm{H} \\
& \text { c. } \mathrm{R}_{2}=\mathrm{R}_{4}=\mathrm{OCH}_{3}, \mathrm{R}_{3}=\mathrm{OH}, \mathrm{R}_{1}=\mathrm{H} \\
& \text { d. } \mathrm{R}_{2}=\mathrm{OCH}_{3}, \mathrm{R}_{3}=\mathrm{OH}, \mathrm{R}_{1}=\mathrm{R}_{4}=\mathrm{H} \\
& \text { e. } \mathrm{R}_{2}-\mathrm{R}_{3}=-\mathrm{OCH}_{2} \mathrm{O}-, \mathrm{R}_{1}=\mathrm{R}_{4}=\mathrm{H} \\
& \text { f. } \mathrm{R}_{1}=\mathrm{R}_{3}=\mathrm{OCH}_{3}, \mathrm{R}_{2}=\mathrm{R}_{4}=\mathrm{H} \\
& \text { g. } \mathrm{R}_{3}=\mathrm{OCH}_{3}, \mathrm{R}_{1}=\mathrm{R}_{2}=\mathrm{R}_{3}=\mathrm{H}
\end{aligned}
$$<smiles>COc1ccc(OC)c(/C=C/c2ccc3ccc(=O)oc3c2)c1</smiles><smiles>COc1cccc(/C=C/c2ccc3ccc(=O)oc3c2)c1OC</smiles><smiles>COc1cc(/C=C/c2ccc3ccc(=O)oc3c2)cc(OC)c1O</smiles><smiles>COc1cc(/C=C/c2ccc3ccc(=O)oc3c2)ccc1O</smiles><smiles>O=c1ccc2ccc(/C=C/c3ccc4c(c3)OCO4)cc2o1</smiles>

Scheme 2. Synthetic pathway to 7-styrylcoumarins

The structures of all compounds have been established by a combined study of IR, ESI-MS, ${ }^{1} \mathrm{H}-$ NMR, ${ }^{13} \mathrm{C}-\mathrm{NMR}$ and COSY spectra. IR spectra exhibited characteristic absorption peaks corresponding to $\mathrm{C}=\mathrm{O}, \mathrm{C}=\mathrm{C}, \mathrm{C}=\mathrm{C}_{\mathrm{Ar}}, \mathrm{C}-\mathrm{O}-\mathrm{C}$, and $\mathrm{C}-\mathrm{H}_{\mathrm{Ar}}$. ESI-MS spectra showed characteristic $[\mathrm{M}+\mathrm{H}]^{+}$peaks corresponding to their molecular weights. The assignments of all the signals to individual $\mathrm{H}$ or $\mathrm{C}$-atoms have been performed on the basis of typical $\delta$-values and $J$-constants. The ${ }^{1} \mathrm{H}-\mathrm{NMR}$ spectra of 3-styrylcoumarins dissolved in $\mathrm{CDCl}_{3}$ showed signals of $-\mathrm{CH}_{3}(\sim 2.60 \mathrm{ppm})$, $\mathrm{OCH}_{2^{-}}(\sim 4.00 \mathrm{ppm}), \mathrm{H}-\mathrm{C}=\mathrm{C}-\mathrm{H}_{\text {trans }}(\sim 7.00$ and $7.60 \mathrm{ppm})$ and 7-styrylcoumarins showed signals of $\mathrm{H}-\mathrm{C}=\mathrm{C}-\mathrm{H}_{\text {cis }}(\sim 6.50$ and $8.00 \mathrm{ppm})$ and $\mathrm{H}-\mathrm{C}=\mathrm{C}-\mathrm{H}_{\text {trans }}(\sim 7.15$ and $7.40 \mathrm{ppm}) .{ }^{13} \mathrm{C}-\mathrm{NMR}$ spectra 3-of styrylcoumarins showed at around 14, 68, 118, 135 and $161 \mathrm{ppm}$, corresponding to $-\mathrm{CH}_{3},-\mathrm{CH}_{2} \mathrm{O}-$, $\mathrm{H}-\mathrm{C}=\mathrm{C}-\mathrm{H}_{\text {trans }}$, and $\mathrm{C}=\mathrm{O}$, respectively. 7-Styrylcoumarins showed signals around 116, 145, 124, 132 and 160 ppm, corresponding to $\mathrm{H}-\mathrm{C}=\mathrm{C}-\mathrm{H}_{\mathrm{cis}}, \mathrm{H}-\mathrm{C}=\mathrm{C}-\mathrm{H}_{\text {trans }}$, and $\mathrm{C}=\mathrm{O}$, respectively.

\section{Effect of styrylcoumarins on SW480 and CHO K1 cell viability}

A series of 3- and 7-styrylcoumarin hybrids were previously synthesized, and evaluated against SW480 and CHO-K1 cell lines, in order to asses their effect on the viability, through the trypan blue dye exclusion method. As shown in Figure 3, the activity was time- and concentration-dependent, with a higher cytotoxic effect on SW480 cells in comparison with CHO-K1 cells. Cytotoxicity was reported as $50 \%$ inhibitory concentration $\left(\mathrm{IC}_{50}\right)$ values. 

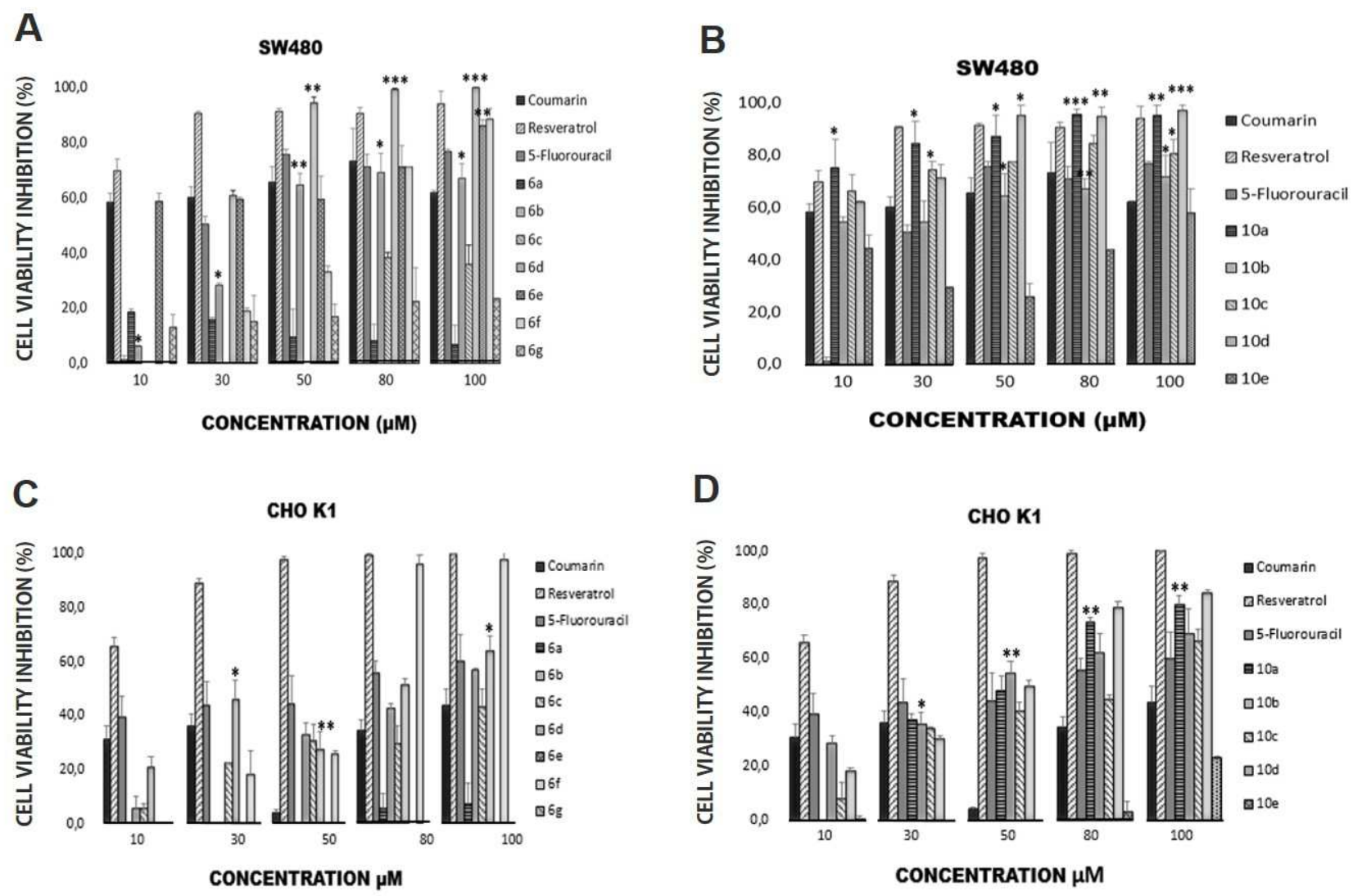

Fig. 3. Effect of 3- and 7-styrylcoumarins on cell viability of SW480 (A and B) and CHO K1 cells (C and D), respectively, 48 hours post-treatment with diferent concentrations of styrylcoumarins $(10-100 \mu \mathrm{M})$. Viable cells were counted and growth inhibition was calculated using $100 \%$ viability of control. Zero values indicate that there was not inhibition at that concentration. Data are presented as the mean $\pm \mathrm{SE}$ of three independent experiments $(* \mathrm{P}<0.05 ; * * \mathrm{P}<0.01 ; * * * \mathrm{P}<0.001)$.

All results regarding to the cytotoxic effect are summarized in Table 1. Among the compounds tested, hybrids $\mathbf{6 a}, \mathbf{6 c}, \mathbf{6 g}$ and 10e exhibited the lowest activity at both 24 and 48 hours after treatment. Besides, excluding compound 10e, the increase of $\mathrm{IC}_{50}$ concentration at 48 hours suggests a loss in the cytotoxic activity of these hybrids. On the other hand, although compound $\mathbf{6 f}$ exhibited good activity against SW480 cancer cell line after 24 hours of treatment $\left(\mathrm{IC}_{50}=23,77 \mu \mathrm{M}\right)$, this effect decreased after 48 hours $\left(\mathrm{IC}_{50}=50,64 \mu \mathrm{M}\right)$ as evidenced by the rise in the $\mathrm{IC}_{50}$. In addition, compound 6 f showed a low selectivity index (24h: 1,$51 ; 48 \mathrm{~h}: 0,9)$. Moreover, compounds 6b and 6d exhibited moderate activity against SW480 cell line after 24 hours of treatment (6b: $\mathrm{IC}_{50}=40,53 \mu \mathrm{M} ; \mathbf{6 d}: \mathrm{IC}_{50}=49,75 \mu \mathrm{M}$ ), and even though the activity of $\mathbf{6 d}$ improved after 48 hours (6d: $\mathrm{IC}_{50}=26,07 \mu \mathrm{M}$ ), the selectivity index decreased due to increased toxicity in the non-malignant cell line (IS $=24 \mathrm{~h}: 5,27 ; 48 \mathrm{~h}: 2,7$ ). The opposite occurred with compounds 10a and 10b which 
displayed the highest activity after 24 hours of treatment ( $\mathrm{IC}_{50}=0,10$ and $0,031 \mu \mathrm{M}$, respectively), but decreased after 48 hours ( $\mathrm{IC}_{50}=0,68$ and 7,92 $\mu \mathrm{M}$, respectively). Despite the loss in the activity and selectivity of these two compounds over time, it was worthwhile to include them in further studies since they were more potent than the lead and standard compounds at lowest concentrations and time. When compound 10c was tested at 24 hours, it exhibited higher toxicity against the nonmalignant cell $\left(\mathrm{IC}_{50-\mathrm{CHOK} 1}=4,66 \mu \mathrm{M} ; \mathrm{IC}_{50-\mathrm{SW} 480}=9,40 \mu \mathrm{M}\right)$ than the malignant one. However, after 48 hours of treatment the cytotoxicity varied in a significant way for both cell lines $\left(\mathrm{IC}_{50-\mathrm{CHOK} 1}=\right.$ $\left.68,63 \mu \mathrm{M} ; \mathrm{IC}_{50-\mathrm{SW} 480}=1,01 \mu \mathrm{M}\right)$, being these values even better than coumarin and 5-FU (IC50$\mathrm{SW}_{480}=3.91 \mu \mathrm{M} ; \mathrm{IC}_{50-\mathrm{SW} 480}=35,43 \mu \mathrm{M}$, respectively). This fact was also reflected in the increase of the selectivity index for compound 10c. Finally, compounds 6e and 10d showed better activity against SW480 cells $\left(\mathbf{6 e}: \mathrm{IC}_{50-24 \mathrm{~h}}=11,15 \mu \mathrm{M}, \mathrm{IC}_{50-48 \mathrm{~h}}=6,92 \mu \mathrm{M} ; \mathbf{1 0 d}: \mathrm{IC}_{50-24 \mathrm{~h}}=5,47 \mu \mathrm{M}, \mathrm{IC}_{50-}\right.$ $48 \mathrm{~h}=5,33 \mu \mathrm{M})$ in comparison with the non-malignant cell line $\left(6 \mathbf{e}: \mathrm{IC}_{50-24 \mathrm{~h} / 48 \mathrm{~h}}=>3000 \mu \mathrm{M} ; \mathbf{1 0 d}\right.$ : $\mathrm{IC}_{50}$ $\left.24 \mathrm{~h}=9,48 \mu \mathrm{M}, \mathrm{IC}_{50-48 \mathrm{~h}}=38,14 \mu \mathrm{M}\right)$ and the activity was kept over time. Once again, these two compounds exhibited higher activity than the lead and standard compounds, which shows that the improvement in the activity and selectivity could be due to a synergistic action of the lead compounds when they are linked to form a single structure in the hybrid. This study is consistent with the findings of Belluti et al (2010) which found similar results in the activity of some stilbenecoumarin hybrids when they were tested in other cancer cell lines (A431 epidermoid carcinoma, JR8 melanoma cell and H460 non-small cell lung cancer). 
Table 1 Cytotoxic effect $(\mu \mathrm{M})$ of styrylcoumarins on SW480 and CHO K1 cell lines

\begin{tabular}{|c|c|c|c|c|c|c|}
\hline \multirow[b]{2}{*}{ Compounds } & \multicolumn{3}{|c|}{24 hours } & \multicolumn{3}{|c|}{48 hours } \\
\hline & $\begin{array}{l}\mathrm{IC}_{50}(\mu \mathrm{M}) \\
\text { CHO cells }\end{array}$ & $\begin{array}{c}\mathrm{IC}_{50}(\boldsymbol{\mu M}) \\
\mathrm{SW} 480 \text { cells }\end{array}$ & SI & $\begin{array}{l}\mathrm{IC}_{50}(\mu \mathrm{M}) \\
\text { CHO cells }\end{array}$ & $\begin{array}{c}\mathrm{IC}_{50}(\boldsymbol{\mu M}) \\
\text { SW480 cells }\end{array}$ & SI \\
\hline $6 a$ & $>3000$ & 61,81 & $>40$ & $>3000$ & $>3000$ & $>1$ \\
\hline $6 b$ & 66,26 & 40,53 & 1,63 & 110,07 & 45,50 & 2,4 \\
\hline $6 c$ & 419,69 & 109,49 & 3,83 & 213,10 & 576,91 & 0,4 \\
\hline $6 \mathrm{~d}$ & 262,41 & 49,75 & 5,27 & 69,81 & 26,07 & 2,7 \\
\hline $6 e$ & $>3000$ & 11,15 & $>200$ & $>3000$ & 6,92 & $>400$ \\
\hline $6 f$ & 35,88 & 23,77 & 1,51 & 43,61 & 50,64 & 0,9 \\
\hline $6 \mathrm{~g}$ & $>3000$ & 295,09 & $>10$ & $>3000$ & $>3000$ & $>1$ \\
\hline $10 \mathrm{a}$ & 254,18 & 0,10 & 2534,80 & 43,90 & 0,68 & 64,8 \\
\hline $10 \mathrm{~b}$ & 4,55 & 0,031 & 147,26 & 40,86 & 7,92 & 5,2 \\
\hline $10 \mathrm{c}$ & 4,66 & 9,40 & 0,50 & 68,63 & 1,01 & 67,8 \\
\hline 10d & 9,48 & 5,47 & 1,73 & 38,14 & 5,33 & 7,2 \\
\hline $10 \mathrm{e}$ & 1546,22 & 2020,28 & 0,16 & $>3000$ & 92,88 & $>30$ \\
\hline Coumarin & 2541,85 & 32,12 & 79,13 & 1251,66 & 3,91 & 319,7 \\
\hline Resveratrol & 9,00 & 8,90 & 1,01 & 2,87 & 0,89 & 3,2 \\
\hline 5-Fluorouracil & 90,99 & 61,68 & 1,48 & 47.63 & 35,43 & 1.34 \\
\hline
\end{tabular}

All compounds that exhibited good activity and selectivity with the trypan blue method $(\mathbf{6 b}, \mathbf{6 d}, \mathbf{6 e}$, 10a, 10b, 10c and 10d) were tested in order to determine their antiproliferative effect with sulforhodamine B assay, which is used for cell density determination based on the measurement of cellular protein content. After comparing each treatment with the control, results indicated that the activity was time- and concentration-dependent. Among the results obtained, hybrids $\mathbf{6 e}, \mathbf{1 0 c}$ and 10d displayed significant antiproliferative activity even at low concentrations $\left(\mathrm{IC}_{50}\right.$ values lower than $12 \mu \mathrm{M})$ from day 4 of the treatment, while hybrid $\mathbf{6 d}$ required a higher concentration $(>20 \mu \mathrm{M})$ 
to exert a significant antiproliferative effect in the same time. Furthermore, molecule $\mathbf{6 b}$ required less time ( 2 days) but a higher concentration $(50 \mu \mathrm{M})$ to display a significant activity $(\mathrm{p} \leq 0.05)$. On the other hand, none of the concentrations evaluated for hybrids 10a and 10b caused cell growth inhibition over time (Fig. 4).
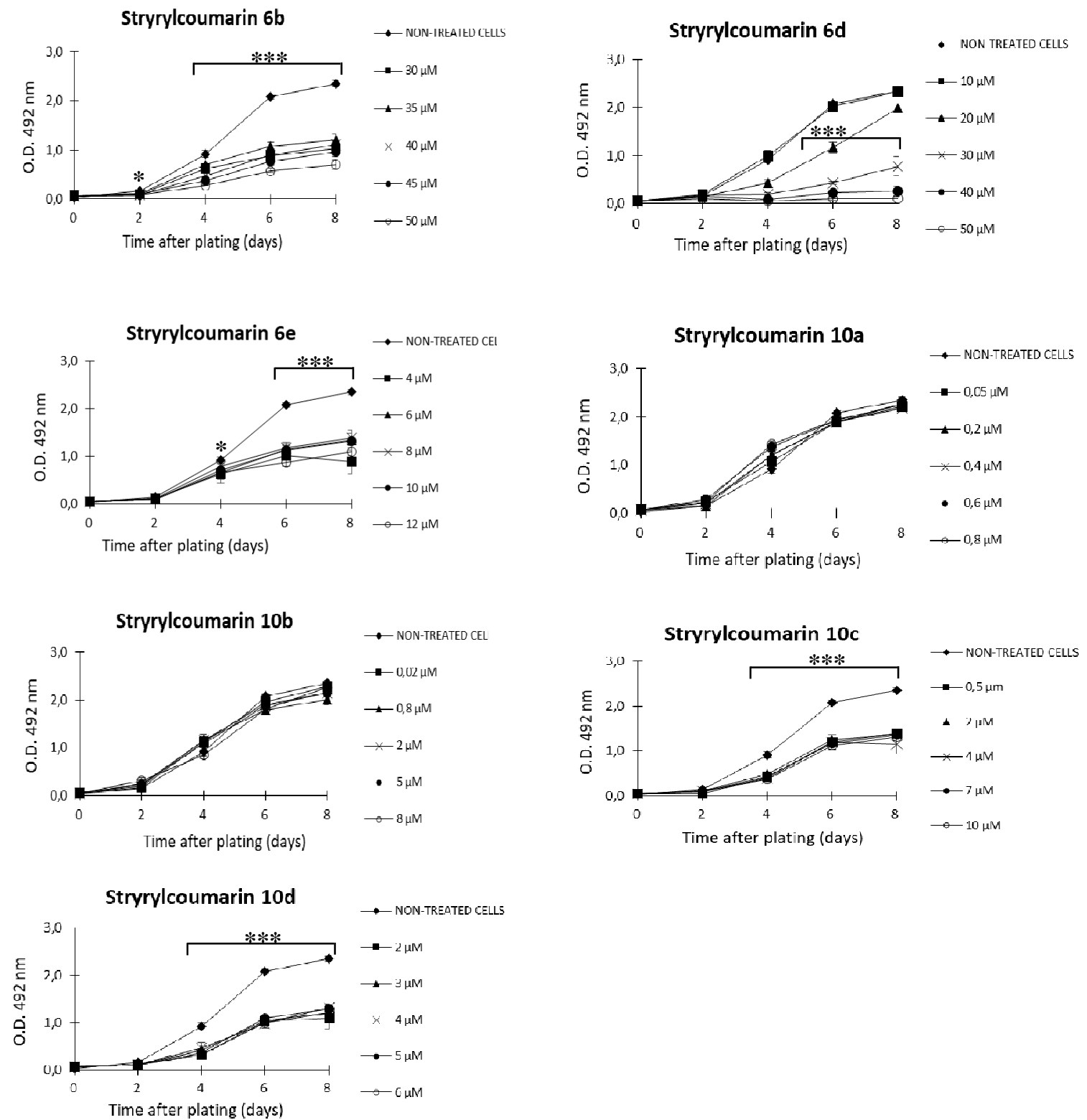

Fig. 4. Antiproliferative effect of styrylcoumarins on SW480 colon cancer cell growth. Data are presented as the mean $\pm \mathrm{SE}$ of at least three independent experiments $(* \mathrm{P}<0.05 ; * * \mathrm{P}<0.01 ; * * * \mathrm{P}<0.001)$. Optical Density (O.D.) is directly proportional to cell mass of adherent cells.

On a structure-activity relationship, it is interesting to note that in general, the position of styryl group is important for the activity. Thus, when series $\mathbf{6}$ and $\mathbf{1 0}$ are compared a higher prevalence in the toxicity against SW480 cells, with better selectivity indexes for $\mathbf{1 0}$ series, is observed (see compounds 10a-10d). For instance, when compounds $6 \mathbf{f}$ and 10c, bearing the 4-hydroxy-3,5- 
dimethoxystyryl motif, are compared a divergence of activities is observed, being the hybrid 10c the one which is able to preserve the best activity over time. On the other hand, we found that oxygenated positions in 3,4 as well as 2,5 positions were determinant for the activity. These results are similar to the ones obtained by Belluti et al. (2010) on H460 cells when they were treated with 7-methoxycoumarin having 3,5-disubstitution pattern in trans-vinylbenzene moiety. However, we noticed that the presence of a hydroxyl group in 4-position was necessary to preserve the activity through time (6d, 10c and 10d vs 10a) (See Figure 4). The effect of the hydroxyl groups may be due to a better molecular recognition ability towards the target bioreceptors upon hydrogen bond formation (Patrick, 2013). Thus, the presence of specific substituents in certain positions proved to be crucial in endowing the compounds with inhibitory effect and antiproliferative. The 4-methoxy and methylendioxy group were detrimental, since the presence of these groups in compounds $\mathbf{6 a}$, $\mathbf{6 g}$, and 10e resulted in a decrease of activity.

\section{Conclusions}

Twelve hybrid molecules combining styryl and coumarin motifs have been designed and synthesized and their antiproliferative activity has been measured against SW480 and CHO-K1 cell lines. Among the tested compounds, hybrids 6e, 10c and 10d exhibited the highest activity which make them promising candidates for further studies. The SAR analysis showed that styryl group in the position 7 and the presence of hydroxyl groups in position 4 or methoxy groups in 2,5 and 3,4positions were determinant for their biological activity.

\section{Acknowledgments}

The authors thank Universidad de Antioquia (grant CODI 2014-808) for financial support.

\section{Conflict of interest}

The authors declare that they have no conflict of interest. 


\section{References}

Adhikari MV, Samant, SD (2002) Sonochemical bromination of acetophenones using ptoluenesulfonic acid N-bromosuccinimide. Ultrasonics Sonochemistry 9:107-111

Agarwal R. Synthesis \& biological screening of some novel coumarin derivatives (2000) Biochem Pharmacol. 6:1042-1051

Ankleka KY, Lakkannavar CD, Kulkarni GM, Kulkarni MV (2003) Synthesis, spectral studies and biological evaluation of some new 4-substituted coumarins. Ind J Chem section B, 42: 15481550

Arango V, Robledo SM, Seón-Méniel B, Figadere B, Cardona W, Sáez J, Otálvaro F (2010) Coumarins from Galipea panamensis and Their Activity against Leishmania panamensis. J Nat Prod 73:1012-1014

Arnold M, Sierra MS, Laversanne M, Soerjomataram I, Jemal A, Bray1 F (2016) Global patterns and trends in colorectal cancer incidence and mortality, Gut 0:1-9.

Athar M, Back JH, Kopelovich L, Bickers DR, Kim AL (2009) Multiple molecular targets of resveratrol: anti-carcinogenic mechanisms. Arch Biochem Biophys 486:95-102

Baur JA, Sinclair DA (2006) Therapeutic potential of resveratrol: the in vivo evidence. Nat Rev Drug Discov 5:493-506

Bellina F, Guazzelli N, Lessi M, Manzini C (2015) Imidazole analogues of resveratrol: synthesis and cancer cell growth evaluation. Tetrahedron 71: 2298-2305

Belluti F, Fontana G, Bo LD, Carenini N, Giommarelli C, Zunino F (2010) Design, synthesis and anticancer activities of stilbene-coumarin hybrid compounds: Identification of novel proapoptotic agents. Bioorg Med Chem 18: 3543-3550

Borriello A, Bencivenga D, Caldarelli I, Tramontano A, Borgia A, Zappia V, Della Ragione F (2014) Resveratrol:from basic studies to bedside. Cancer Treat Res 59:167-184

Budzisz E, Brzezinska E, Krajewska U, Rozalski M (2003) Cytotoxic effects, alkylating properties and molecular modelling of coumarin derivatives and their phosphonic analogues. Eur J Med Chem 38:597-603

Cano M, Rojas C, Hidalgo W, Sáez J, Gil J, Schneider B, Otálvaro F (2013) Improved synthesis of 4-phenylphenalenones: the case of isoanigorufone and structural analogs. Tetrahedron Lett $54: 351-354$

Egan D, James P, Cooke D, O'Kennedy R (1997) Studies on the Cytostatic and Cytotoxic Effects and Mode of Action of 8-Nitro-7-Hydroxycoumarin. Cancer Lett 118:201-211 
Gacche R, Jadhav S (2012) Antioxidant Activities and Cytotoxicity of Selected Coumarin Derivatives: Preliminary Results of a StructureeActivity Relationship Study Using Computational Tools. J Exp Clin Med 4:165-169

Globocan (2012) Estimated Cancer Incidente, Mortality and Prevalence Worldwide in 2012. International Agency for Research on Cancer, World Health Organisation. http://globocan.iarc.fr/Pages/fact_sheets_cancer.aspx

Gordo J, Avo J, A. Parola AJ, Lima JC, Pereira A, S. Branco PS (2011) Convenient Synthesis of 3Vinyl and 3-Styryl Coumarins. Org Lett 13:5112-5115

Gülçin I (2010) Antioxidant properties of resveratrol: A structure-activity insight. Innov Food Sci Emerg Technol. 11: 210-218

Kashman Y, Gustafson KR, Fuller WR, Cardellina JH, McMahon JB, Currens MJ, Buckheit RW Jr, Huqhes SH, Cragg GM, Boyd, M.R (1992) The calanolides, a novel HIV-inhibitory class of coumarin derivatives from the tropical rainforest tree, Calophyllum lanigerum. J Med Chem 35: $2735-2743$

Kawaii S, Tomono Y, Ogawa K, Sugiura M, Yano M, Yoshizawa Y (2001) The anti-proliferative effect of coumarins on several cancer cell lines. Anticancer Res 21:917-923

Kedzierski L, Curtis J, Kaminska M, Jodynis Liebert J, Murias M (2007) In vitro antileishmanial activity of resveratrol and its hydroxylated analogues against Leishmania major promastigotes and amastigotes. Parasitol Res 102: 91-97

Kumar D, Raj KK, Malhotra SV, Diwan S. Rawat DS (2014) Synthesis and anticancer activity evaluation of resveratrol-chalcone conjugates. Med Chem Commun 5: 528-535

Lamy V, Roussi S, Chaabi M, Gossé F, Schall N, Lobstein A, Raul F (2007) Chemopreventive effects of lupulone, a hop b-acid, on human colon cancer-derived metastatic SW620 cells and in a rat model of colon carcinogenesis. Carcinogenesis 28:1575-1581

Madari H, Panda D, Wilson L, Jacobs RS (2003) Dicoumarol: A unique microtubule stabilizing natural product that is synergistic with taxol. Cancer Res 63:1214-1220

Maldonado ME, Bousserouel S, Gossé F, Minker C, Lobstein A, Raul F (2009) Differential Induction of Apoptosis by Apple Procyanidins in TRAIL-Sensitive Human Colon Tumor Cells and Derived TRAIL-Resistant Metastatic Cells. J. Cancer Mol 5: 21-30

Manhas, M, Ganguly S, Mukherjee S, Jain A (2006) Microwave initiated reactions: Pechmann coumarin synthesis, Biginelli reaction, and acylation. Tetrahedron Lett 47: 2423-2425

Martin E, Kellen-Yuen C (2007) Microwave-Assisted Organic Synthesis in the Organic Lab: A Simple, Greener Wittig Reaction. J Chem Ed 84:2004-2006

Martins S, Branco P, Pereira A (2012) An Efficient Methodology for the Synthesis of 3-Styryl Coumarins. J Braz Chem Soc 23: 688-693 
Mayur YC, Peters GJ, Prasad VV, Lemo C, Sathish NK (2009) Design of New Drug Molecules to be Used in Reversing Multidrug Resistence in Cancer Cells. Curr Cancer Drug Targets 9:298306

McQuade RM, Bornstein JC, Nurgali K (2014) Anti-Colorectal Cancer Chemotherapy-Induced Diarrhoea: Current Treatments and Side-Effects. Int J Clin Med 5: 393-406.

Meunier B (2008) Hybrid molecules with a dual mode of action: dream or reality? Acc Chem Res. 41: 69-77

Neckers L, (2002) Hsp90 inhibitors as novel cancer chemotherapeutic agents. Trends Mol Med. 8:S55-61

Otero E, Vergara S, Robledo SM, Cardona W, Carda M, Vélez ID, Rojas C, Otálvaro F (2014) Synthesis, Leishmanicidal and Cytotoxic Activity of Triclosan-Chalcone, Triclosan-Chromone And Triclosan-Coumarin Hybrids. Molecules 19:13251-13266

Patrick GL (2013) An Introduction to Medicinal Chemistry, fifth ed., Oxford University Press, pp. 1-14

Paul K, Bindal S, Luxami V (2013) Synthesis of new conjugated coumarin-benzimidazole hybrids and their anticancer activity. Bioorg Med Chem Lett 23: 3667-3672

Pérez JM, Maldonado ME, Rojano BA, Alzate F, Sáez J, Cardona W (2014). Comparative Antioxidant, Antiproliferative and Apoptotic Effects of Ilex laurina and Ilex paraguariensis on Colon Cancer Cells. Trop J Pharm Res 13: 1279-1286

Pierson J-T, Dumetre A, Hutter S, Delmas F, Laget M, Finet J-P , Azas N, Combes S (2010) Synthesis and antiprotozoal activity of 4-arylcoumarins. Eur J Med Chem 45:864-869

Pointet AL, Taieb J (2017) Cáncer de colon. EMC - Tratado de medicina 21:1-7

Resch M, Steigel A, Chen ZL, Bauer R (1998) 5-Lipoxygenase and cyclooxygenase-1 inhibitory active compounds from Atractylodes lancea. J Nat Prod 61: 347-350

Riveiro ME, Vazquez R, Moglioni A, Gomez N, Baldi A, Davio C, Shayo C (2008) Biochemical mechanisms underlying the proapoptotic activity of 7,8-dihydroxy-4-methylcoumarin in human leukemic cells. Biochem Pharmacol 75:725-736

Sabrina A, Horbach R, Deising HB, Siewert B, Csuk R (2011) Synthesis and antimicrobial activity of (E) stilbene derivatives. Bioorg Med Chem 19:5155-5166

Sashidhara KV, Kumar A, Kumar M, Sarkar J, Sinha S (2010) Synthesis and in vitro evaluation of novel coumarin-chalcone hybrids as potential Anticancer agents. Bioorg Med Chem Lett 20: 7205-7211

Shen W, Mao J, Sun J, Sun M, Zhang C (2013) Synthesis and biological evaluation of resveratrolcoumarin hybrid compounds as potential antitumor agents. Med Chem Res 22:1630-1640

Simpson CJ, Fitzhenry MJ, Stamford NP (2005) Preparation of vinylphenols from 2- and 4- 
hydroxybenzaldehydes. Tetrahedron Lett 46:6893-6896

Solomon VR, Hu C, Lee H (2009) Hybrid pharmacophore design and synthesis of isatinbenzothiazole analogs for their anti-breast cancer activity. Bioorg Med Chem 17:7585-7592

Symeonidis T, Chamilos M, Hadjipavlou-Litina DJ, Kallitsakis M, Litinas KE (2009) Synthesis of hydroxycoumarins and hydroxybenzo[f]- or [h]coumarins as lipid peroxidation inhibitors. Bioorg Med Chem Lett 19:1139-1142

Temraz S, Mukherji D, Shamseddine A (2013). Potential targets for colorectal cancer prevention Int J Mol Sci. 14:17279-17303

Tsogoeva SB (2010) Recent Progress in the Development of Synthetic Hybrids of Natural or Unnatural Bioactive Compounds for Medicinal Chemistry. Mini Rev Med Chem 10:773-793

Udenigwe CC, Ramprasath VR, Aluko RE, Jones PJ (2008) Potential of resveratrol in anticancer and anti-inflammatory therapy. Nutr Rev 66:445-454

Vilar S, Quezada E, Santana L, Uriarte E, Yanez M, Fraiz N, Alcaide C, Cano E, Orallo F (2006) Design, sysnthesis and vasorelaxant and platelet antiaggregatory activities of coumarinresveratrol hybrids. Bioorg Med Chem Lett 16:257-261

Yang JY, Della Fera MA, Baile CA (2006) Esculetin induces mitochondria-mediated apoptosis in 3T3-L1 adipocytes. Apoptosis 11:1371-1378

Yang J, Liu GY, Dai F, Cao XY, Kang YF, Hu LM, Tang JJ, Li XZ, Li Y, Jin XL, Zhou B (2011) Synthesis and biological evaluation of hydroxylated 3-phenylcoumarins as antioxidants and antiproliferative agents. Bioorg Med Chem Lett 21: 6420-6425

Yee SW, Shah B, Simons C (2005) Synthesis and antimycobacterial activity of 7-O-substituted-4methyl-2H-2-chromenone derivatives vs Mycobacterium tuberculosis, J Enz Inh Med Chem 20: $109-113$

Zhao H, Donnelly AC, Kusuma BR, Brandt GE, Brown D, Rajewski RA, Vielhauer G, Holzbeierlein J, Cohen MS, Blagg BS (2011) Engineering an antibiotic to fight cancer: optimization of the novobiocin scaffold to produce anti-proliferative agents. J Med Chem 9: $3839-3853$ 